Projets

de paysage

\section{Projets de paysage}

Revue scientifique sur la conception et l'aménagement de l'espace

19 | 2018

L'imagerie du paysage

\title{
Les images paysagères autour du thé et de la Chine dans la publicité : quels simulacres de paysage?
}

Landscapes of Tea and of China in Advertising: Imitations of Landscapes

\section{Yun Zhang}

\section{OpenEdition}

\section{Journals}

Édition électronique

URL : http://journals.openedition.org/paysage/371

DOI : $10.4000 /$ paysage.371

ISSN : 1969-6124

\section{Éditeur :}

École nationale supérieure du paysage de Versailles-Marseille, Institut national des sciences appliquées Centre Val de Loire - École de la nature et du paysage, École nationale supérieure d'architecture et de paysage de Bordeaux, École nationale supérieure d'architecture et de paysage de Lille, Agrocampus Angers

Référence électronique

Yun Zhang, "Les images paysagères autour du thé et de la Chine dans la publicité : quels simulacres de paysage? », Projets de paysage [En ligne], 19 | 2018, mis en ligne le 01 décembre 2018, consulté le 28 novembre 2019. URL : http://journals.openedition.org/paysage/371 ; DOI : 10.4000/paysage.371

Ce document a été généré automatiquement le 28 novembre 2019.

Projets de paysage 


\title{
Les images paysagères autour du thé et de la Chine dans la publicité : quels simulacres de paysage?
}

\author{
Landscapes of Tea and of China in Advertising: Imitations of Landscapes
}

\author{
Yun Zhang
}

1 La Chine qui fut le premier pays à découvrir, cultiver et utiliser le théier est aujourd'hui le plus grand producteur de thé au monde ${ }^{1}$ avec une production artisanale en diminution et une part de plus en plus importante de production industrielle, réalisée par de grands producteurs nationaux et des géants internationaux comme Lipton. Les paysages du thé, en tant que paysages emblématiques de la culture chinoise, sont largement utilisés dans la publicité, et sont vendeurs. Ils reflètent non seulement les modes de production agricole mais symbolisent aussi l'environnement géographique et la structure sociale; ils donnent à voir les coutumes locales, forment la mémoire collective et définissent l'appartenance au lieu des acteurs (producteurs, agriculteurs). Ils viennent ainsi inscrire le consommateur dans une Chine traditionnelle tout en le rattachant aux buveurs de thé, nombreux et dispersés partout sur la surface du globe.

Mon propos est d'interroger l'image paysagère du thé chinois sur le marché intérieur : quels paysages sont donnés à voir et en parallèle quelles agricultures ? Peut-on lire des évolutions dans la communication avant et après les réformes économiques chinoises des années 1980 ? Quels concepts mobiliser pour comprendre l'évolution de l'outil communicationnel paysage dans un contexte de mondialisation? J'entends ainsi croiser une réflexion sur le paysage du thé dans différents medias, dont la publicité, et analyser la manière dont il est formalisé et utilisé. J'aurai recours pour mener cette réflexion à plusieurs entrées disciplinaires (agronomie, paysagisme et communication), sachant que le paysage théicole est peu présent dans la littérature scientifique.

3 Les études autour du thé sont très nombreuses en Chine et en Occident. Un des plus anciens ouvrages français sur le thé, la Monographie du thé, écrit en 1843, montre les compositions des thés, ses torréfactions, sa culture et sa valeur médicinale. (Houssaye, 1843). Dans les années 1930 et 1950, plusieurs chercheurs français (Trochain, Goubeaux, 
Guinard, Deuss, etc.) ont publié sur la culture du thé en Indochine, son histoire et sa fabrication. En 1989, Georges Métailié étudie les modes de consommation du thé en France (Métailié, 1989, p. 105-109). Les chercheurs chinois portent également des regards multiples et multidisciplinaires sur le thé : historiques (origine et évolution des théiers, zones de production, etc.), biologiques (qualité du thé, chromatographie en phase gazeuse, etc.), botanistes, économiques, anthropologiques.

Parallèlement, des sinologues et géographes français ont conduit des recherches sur les paysages chinois. Augustin Berque explore la pensée paysagère de l'Orient en la comparant à celle de l'Occident; il analyse les paysages, les jardins et les peintures chinois sous l'angle de la sémiotique et de la phénoménologie (Berque, 1995). Yolaine Escande interroge le paysage à travers la calligraphie, l'inscription et la peinture, ainsi que le terme de «paysage » en chinois (Escande, 2003, 2005, 2012). François Jullien a également abordé, d'abord en linguiste et en philosophe, les différences entre « paysage » en Europe et « Montagne-eau » en Chine (Julien, 2014).

Si le produit thé et le paysage ont donné lieu à de nombreux travaux, peu ont croisé les deux notions. Les quelques recherches sur les paysages agricoles portent plutôt sur les rizières (Bouchery, 1999, p. 133-165 ; Berque, 1980). Il en est de même en Chine avec un intérêt marqué pour la conservation de la biodiversité, les dynamiques du paysage et l'utilisation des sols.

Dans ce premier volet d'études agronomiques ou paysagères, deux publications sont importantes pour ma réflexion. Il s'agit des recherches de l'anthropologue Kunbing Xiao, qui a analysé la structure sociale et l'évolution de la vie des habitants dans la région montagneuse du Fujian de 1644 à 1949 (production de thé Wuyi) (Xiao, 2013). Le livre de Wolfgang Kubin constitue un autre ancrage avec des descriptions concrètes sur les paysages et une étude de l'agriculture dans la littérature classique chinoise (Kubin, 1985).

7 L'autre volet de références est celui de l'image comme outil publicitaire et communicationnel autour des simulacres d'espaces et de marchandises dans la consommation et la communication (Baudrillard, 1996). Plusieurs approches seront utilisées dans cet article : celle de Barthes qui a analysé une publicité de Panzani dans l'article «Rhétorique de l'image » (Barthes, 1964, p. 40-51) ou celles de Mike Crang qui explore les médias en matière de géographie dans son livre Cultural Geography (Crang, 1998). Je reprendrai le concept de «Time-space compression » proposé par Harvey (1989, p. 201-308).

8 Je mènerai cette réflexion en m'appuyant sur un corpus constitué de photographies, de dessins, d'emballages du thé et de la presse imprimée du Fujian en me focalisant sur quelque temps forts de la fin du XIX ${ }^{\mathrm{e}}$ siècle à nos jour ${ }^{2}$, pour qualifier les évolutions et analyser d'éventuels invariants. J'ai également utilisé des écrits documentaires (ouvrages sur le thé répertoriés dans les bibliothèques et dans les archives) et romanesques. Pour les périodes anciennes, les photographies des plantations de thé du Fujian prises par John Thomson à la fin de $\mathrm{XIX}^{\mathrm{e}}$ siècle et les dessins de l'illustrateur britannique Thomas Allom au XIX ${ }^{\mathrm{e}}$ siècle seront mobilisés quand la presse imprimée et les emballages de thé viendront illustrer les périodes récentes. L'objectif de cette recherche est de travailler sur des corpus différents et des sources de dates variées pour montrer les récurrences du paysage du thé et étudier le paysage en tant qu'outil communicationnel. 


\section{L'invention du paysage du thé et de son image}

\section{Le paysage du thé, un paysage sauvage progressivement domestiqué}

Le théier est une plante ligneuse vivace à feuilles persistantes, baptisée Camellia sinensis par Carl von Linné, dans son ouvrage Species plantarum en 1753. La classification botanique du théier au sein du genre Camellia reste toutefois très complexe, tant les espèces et variétés du théier sont nombreuses. Joseph Robert Sealy, qui fait en 1958 une taxonomie du théier, affirme, d'une part, que le genre Camellia se compose de 81 espèces et, d'autre part, que le théier est un arbre ou un arbuste (Sealy, 1958). En 1984, le botaniste chinois Hongda Zhang retient quant à lui 32 espèces et 3 variétés (Zhang, 1984, p. 1-25). Le Larousse agricole distingue plus simplement deux grands types : le type Chine (Camellia sinensis var. sinensis), à feuilles petites et épaisses, et le type Assam (Camellia sinensis var. assamica), à feuilles très grandes et souples. Mais le botaniste chinois Guangtao Li, qui a analysé l'ADN du théier, souligne deux familles: Camellia yunnanensis (arbre, à grandes feuilles), et Camellia sinensis (arbuste ou arbrisseau, à petites feuilles) (LI, 1983, p. 11-16).

Sous la dynastie Han (206 av. J.-C. à 220 ap. J.-C.), le thé est déjà une marchandise ainsi qu'en atteste le «Contrat僮约》 rédigé par Bao Wang en 59 av. J.-C., soit une série d'instructions destinées à son nouveau serviteur. Il s'agit du plus ancien récit sur la production du thé : «Couper finement le poisson frais et le cuire, bouillir l'eau et faire du thé... Prendre le chien et vendre l'oie, puis aller à Wuyang (Sud du Chengdu, Sichuan) pour acheter du bon thé. » Sous la dynastie Tang, Le Classique du thé de Yu Lu énonce pour la première fois les formes de culture du thé : les théiers y sont décrits comme des arbrisseaux d'une hauteur de 0,3 à 0,6 mètre, tels qu'on en trouve en Fujian, en Hunan et à Canton, mais certains théiers sauvages du Yunnan, du Guizhou et du Sichuan peuvent atteindre plusieurs mètres, voire plus de dix mètres (Lu, 2015, p. 43) . Selon les registres de $\mathrm{Yu} \mathrm{Lu}$, les théiers sauvages poussent dans les hautes montagnes et sont entourés d'autres espèces tandis que les théiers cultivés, dont la domestication date au moins du VIII ${ }^{e}$ siècle, sont généralement plantés sur des pentes ensoleillées, là où sont installés les villageois (ibid.).

11 L'évolution des variétés du théier, des espèces sauvages aux cultivars, et surtout de leurs formes, a été étudiée par Wenhuai Chen dans les années 1960 : arbre à l'état sauvage, le théier devient arbuste quand il est cultivé, puis arbrisseau; les feuilles diminuent également de taille et les branches se font plus denses (de monopodial à sympodial); enfin le nombre de poils sur les bourgeons augmente et la période de germination passe de tardive à précoce (Chen, 1964, p. 191-198). On retrouve dans cette généalogie les paysages décrits par Yu Lu.

Les paysages du thé s'accordent aux formes topographiques et aux systèmes agraires des zones où ils sont cultivés (figure 1). Le Fujian est une région montagneuse et côtière: les monts Wuyi se situent au nord-ouest du Fujian avec un paysage typiquement montagnard alors que Fuzhou, à l'est du Fujian, présente un paysage hybride de littoral et de collines. De ce fait, les plantations de thé des deux zones montrent certaines différences. Sur les pentes raides des monts Wuyi, dit relief "Danxia », les plantations de thé sont faites sur des terrasses en pierre, irrégulières et de petite taille (figure 2), $80 \%$ des anciens jardins de thé aux monts Wuyi étaient de 
cette forme. Si la culture en terrasses se retrouve parfois à Fuzhou, l'originalité de cette zone tient à la production de thé au jasmin d'où un double paysage, celui du thé et celui de la culture des fleurs de jasmin. Fuzhou, une ville littorale avec des pentes abruptes vers la mer, montre des paysages verticaux remarquables avec un étagement de la végétation: en altitude la forêt, puis les jardins de thé, à mi-pente une autre zone forestière, et enfin les jardins de jasmin et la rivière (Min, Zhang, 2015, p. 43) (figure 3).

Figure 1. Carte de la province du Fujian

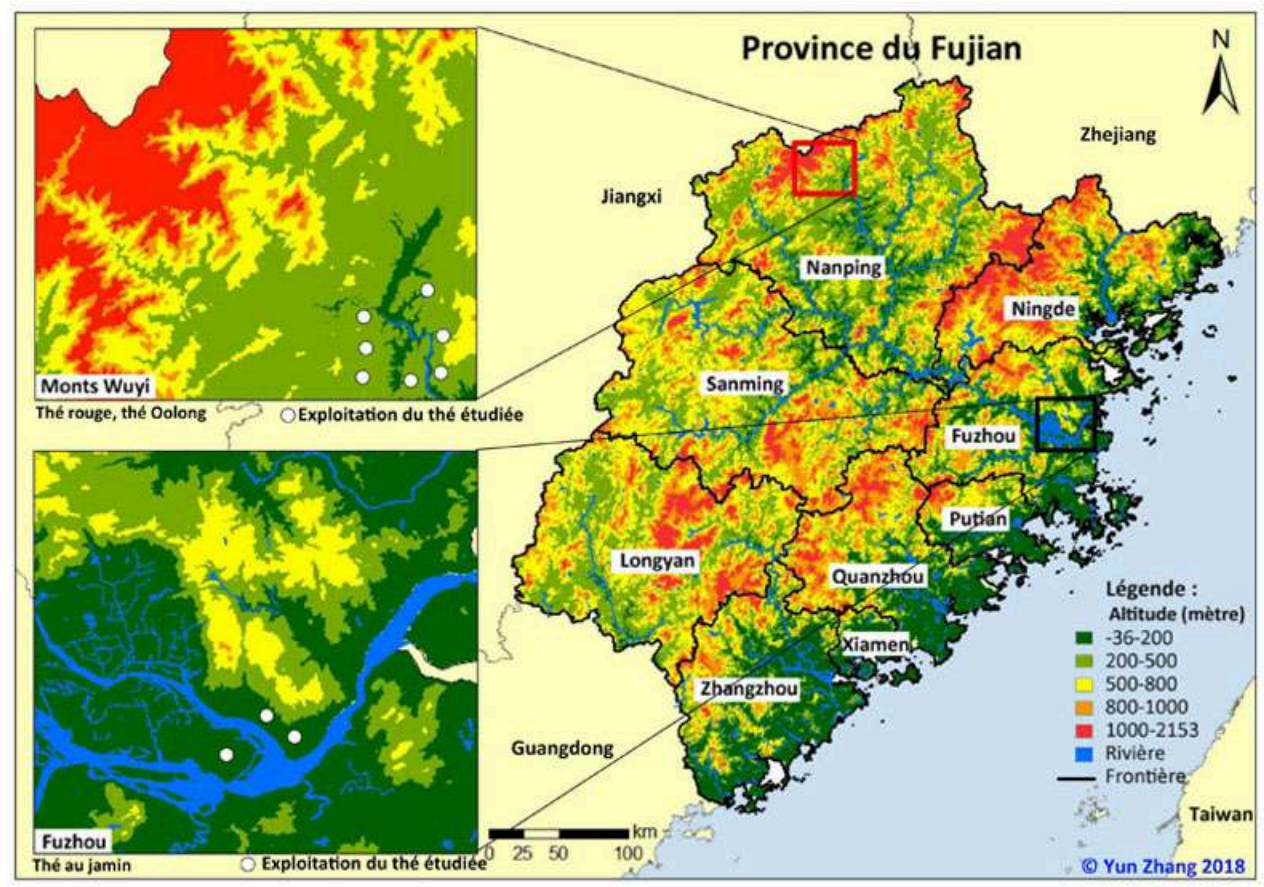

Elle a été réalisée avec Arcgis en octobre 2018. 
Figure 2. Jardin du thé en terrasses de pierre aux monts Wuyi

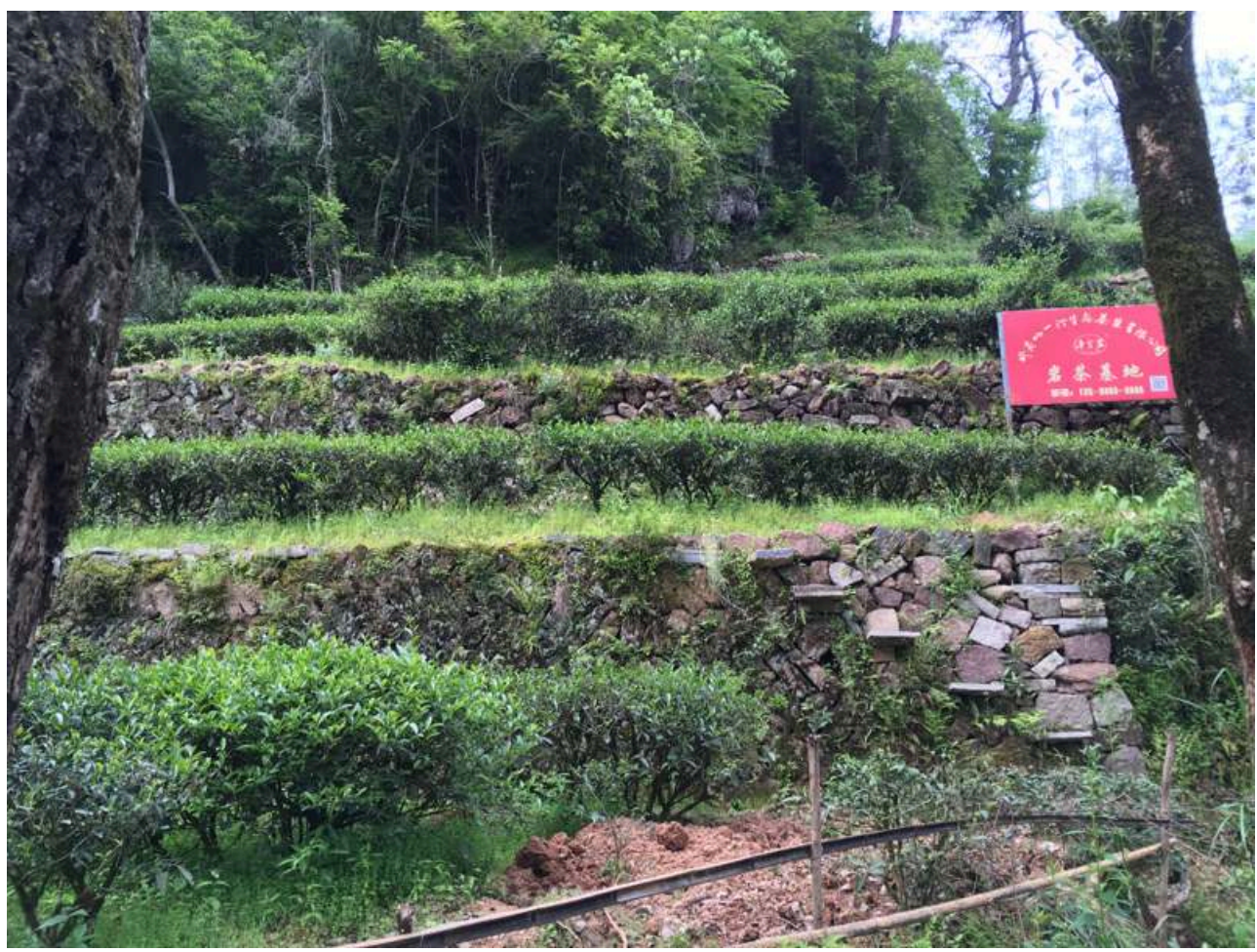

Photographié en mai 2018.

Figure 3. Paysages verticaux à Fuzhou

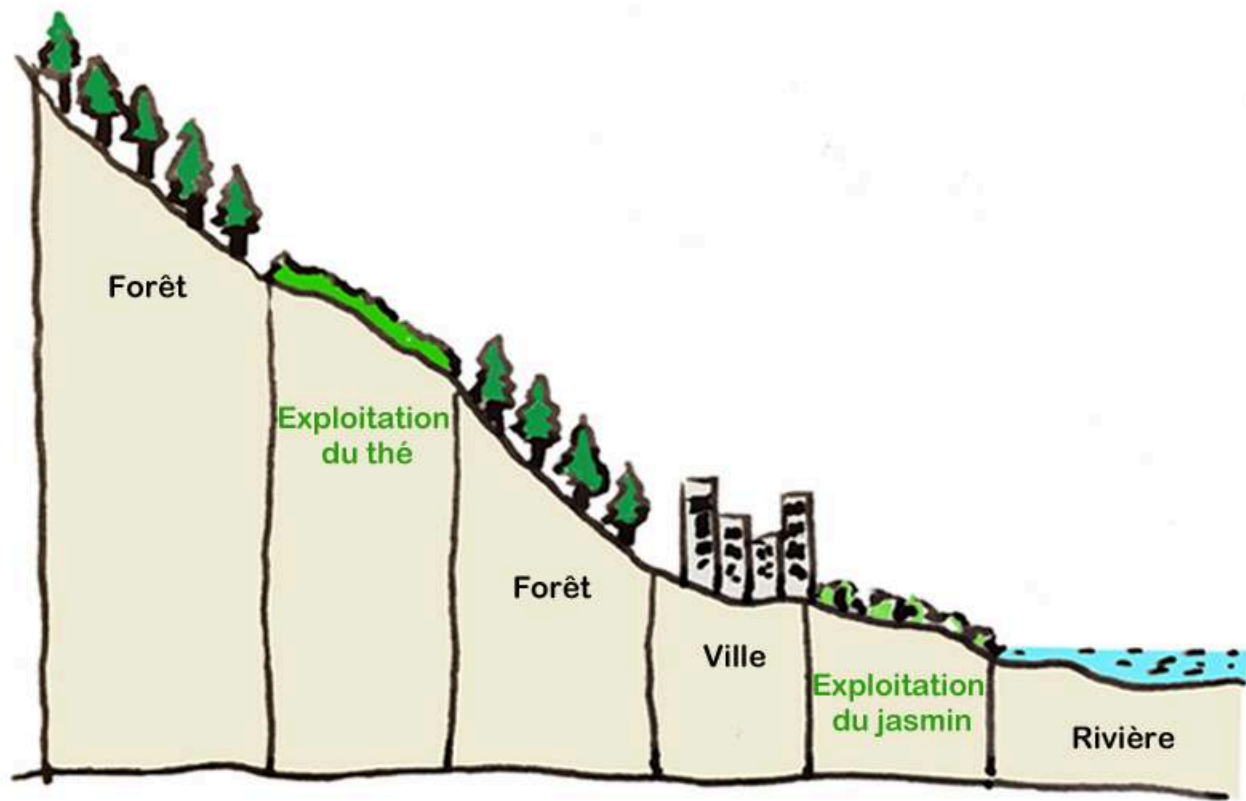

Paysages verticaux à Fuzhou

Source : Min, Zhang, 2015. 
Sur ces terrasses, la culture des théiers s'est faite en touffe jusque dans les années 1950 : chaque arbuste était séparé du suivant de plusieurs centimètres, ce qui est un peu similaire aux vignes de style Gobelet. Les plantations en rang (figure 4) se sont généralisées à partir de cette date mais les agriculteurs ont maintenu une végétation naturelle ou forestière autour des jardins de thé ${ }^{4}$ pour ralentir l'écoulement de l'eau. En outre, les théiers poussent parfois avec d'autres cultures (par exemple les rizières) dans des formes proches de la coltura promiscua. Le paysage du thé présente donc à la fois une image de nature et une image d'anthropisation. L'espacement des rangs est d'environ 1,5 mètre, la distance entre deux théiers d'une même ligne d'environ 33 centimètres et les arbustes sont taillés chaque année. Cette forme régulière fait ressembler ces paysages à ceux de la vigne de forme "guyot double» et "cordon double " (figure 5). Mais les jeunes théiers qui ne sont pas encore taillés ressemblent toujours aux jardins du thé « en touffe » (figure 6).

Figure 4. Jardin moderne, en rang aux monts Wuyi

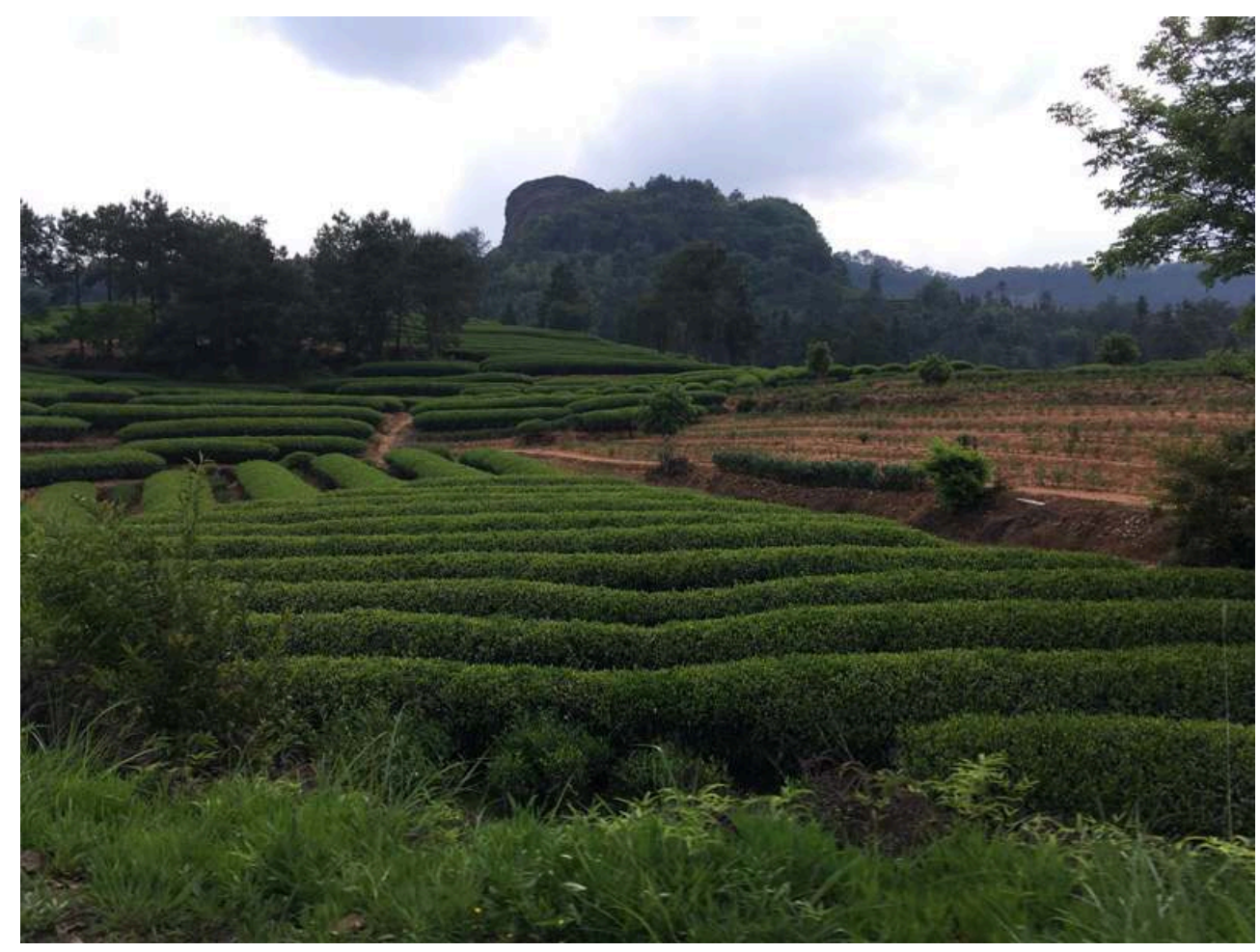

Photographié en mai 2018. 
Figure 5. Taillage du théier
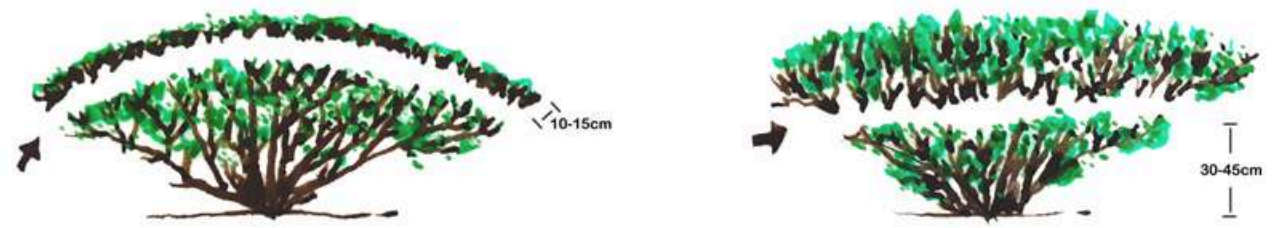

Taillage du théier adulte

Retaillage du vieux théier

Figure 6. Les théiers jeunes

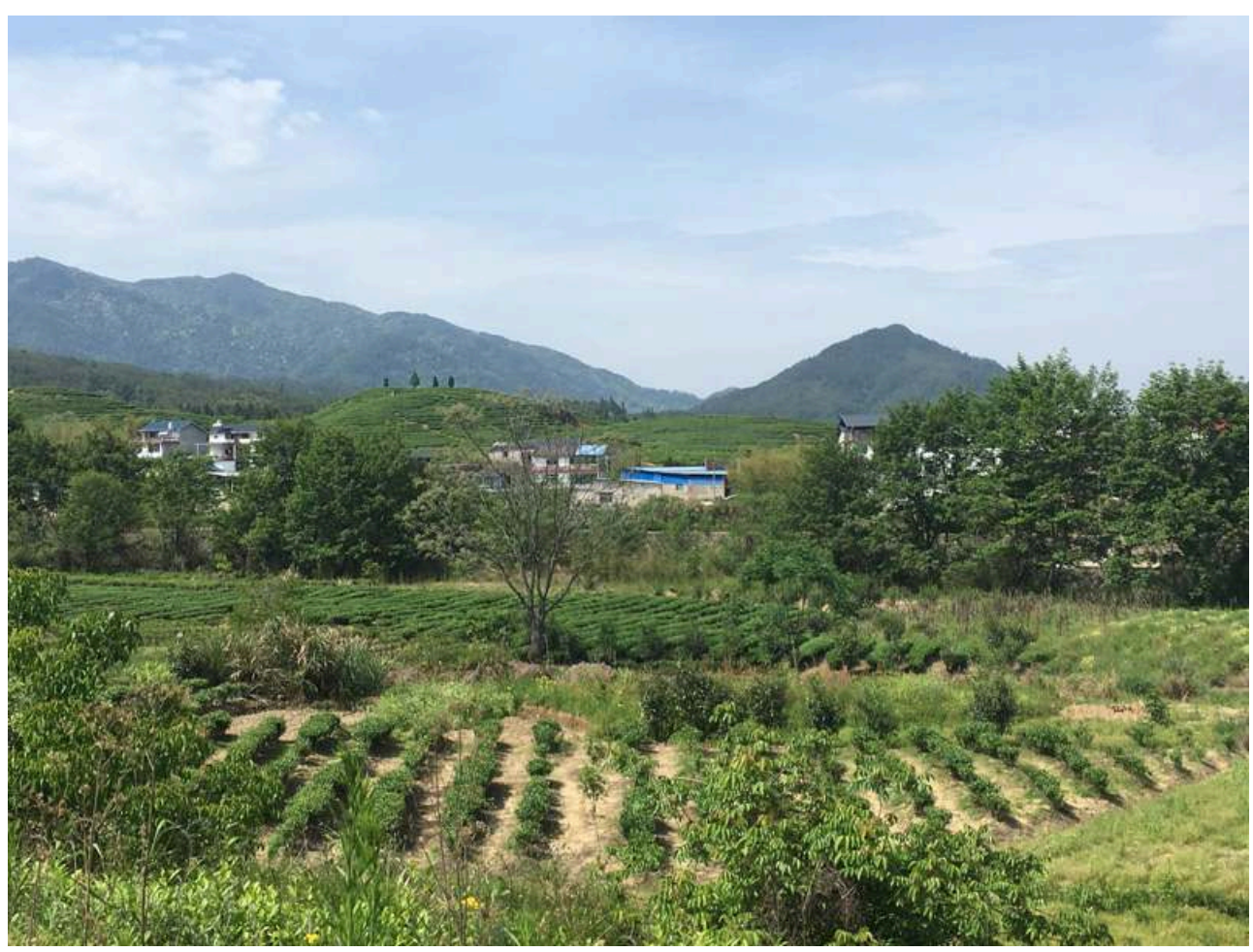

Photographiés en mai 2018 
14 La culture du thé crée donc un paysage profondément humanisé avec des parcelles à flanc de montagnes ou de collines, souvent en terrasses, et des plants réguliers et taillés. Les paysages du thé des monts Wuyi montrent une structure spatiale riche et complexe : certains renvoient à une culture de main-d'œuvre sur de petites parcelles, telles que celles à côté des champs et des maisons, ce qui dans notre imaginaire est synonyme de culture familiale (figure 7) ; parallèlement, certains théiers sauvages dans les hautes montagnes participent de l'image de la nature profonde et soutiennent un imaginaire ancestral (figure 8); enfin les jardins en rang reflètent une certaine modernité (figure 9). Ce paysage hybride révèle un compromis entre la culture traditionnelle, autosuffisante qui alimente la nostalgie moderne et un monde urbanisé et industrialisé.

Figure 7. Petite parcelle du thé à côté de la maison aux monts Wuyi

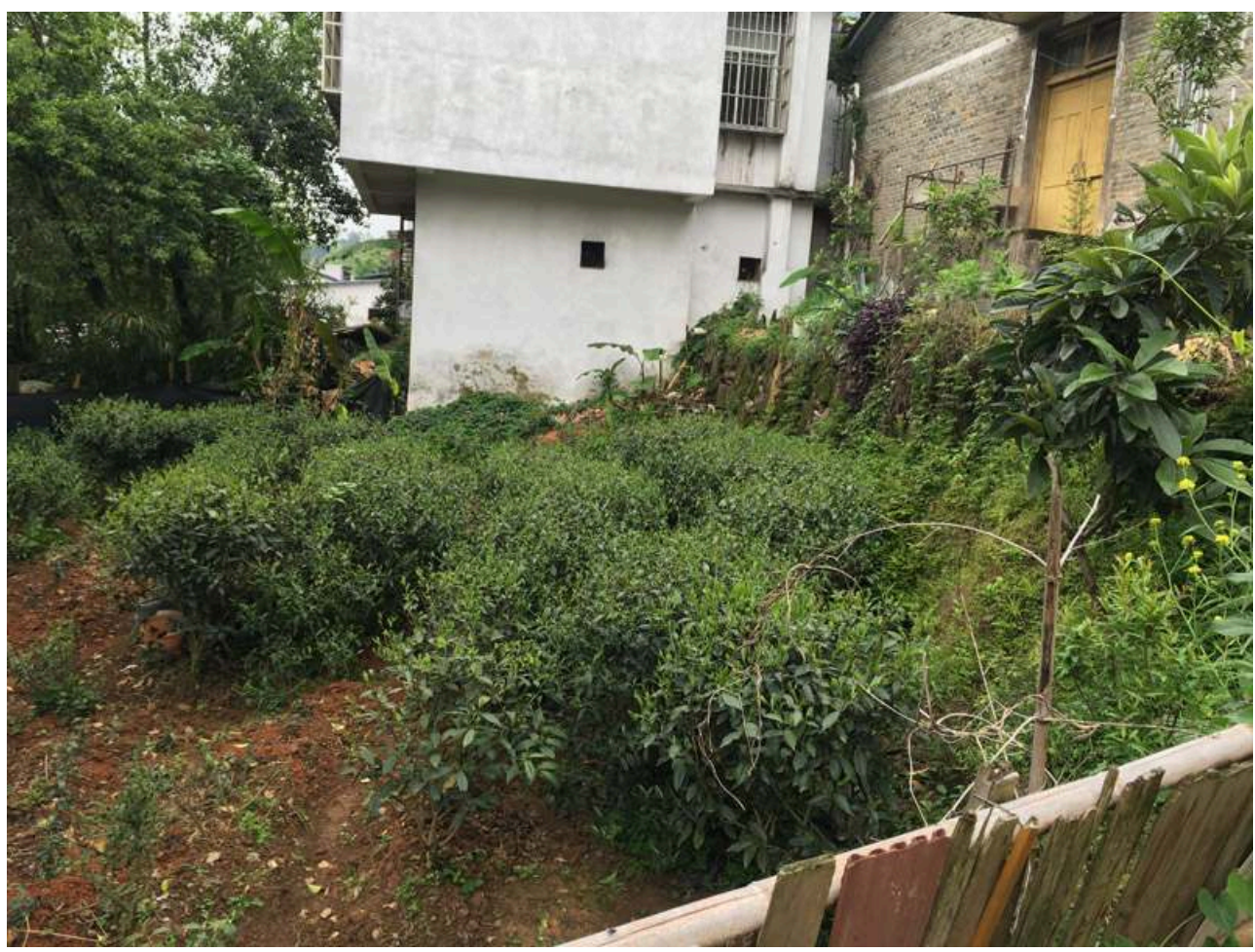

Photographiée en mai 2018. 
Figure 8. Plantation du thé dans la montagne profonde aux monts Wuyi

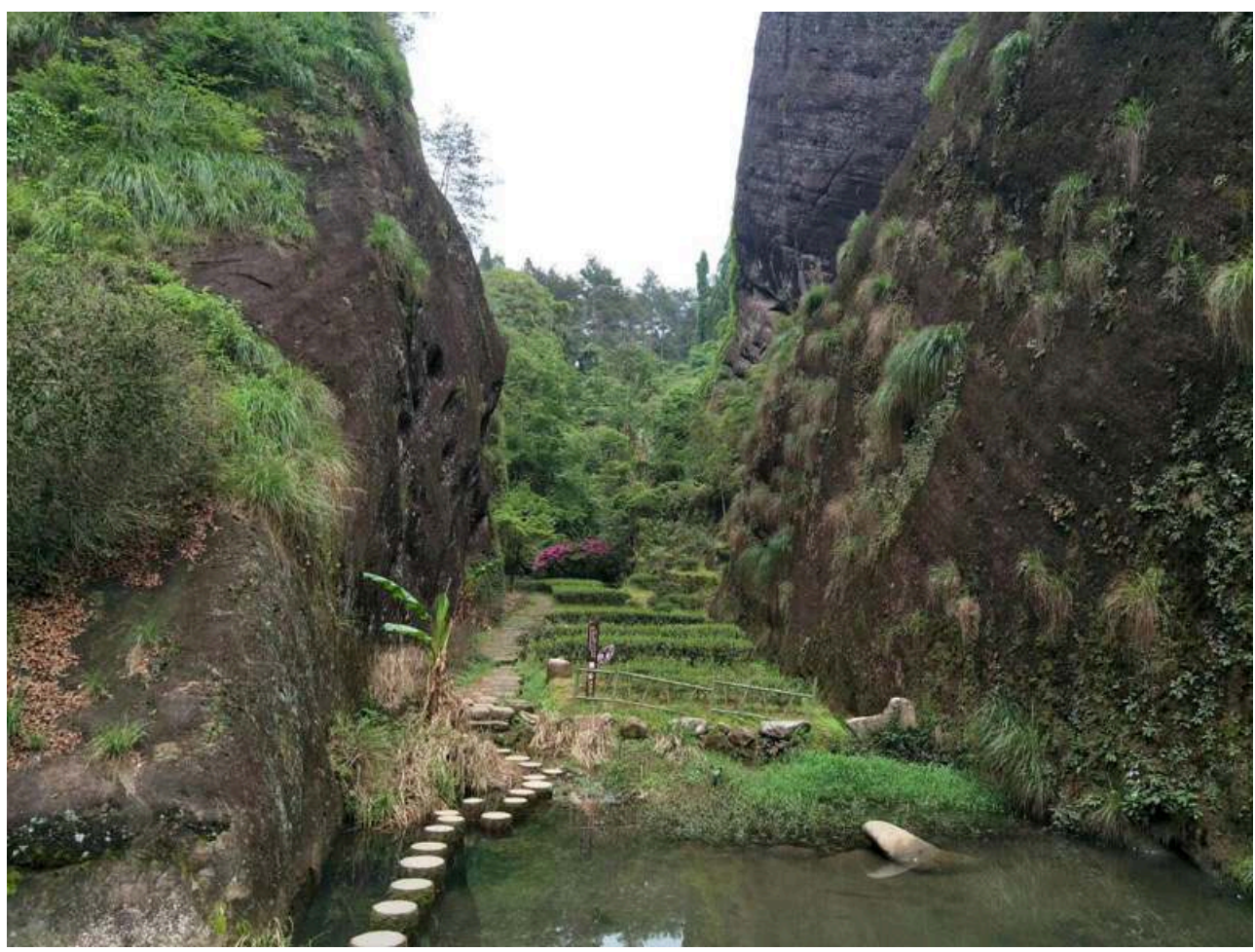

Photographiée en mai 2018.

Figure 9. Plantation du thé à grande échelle

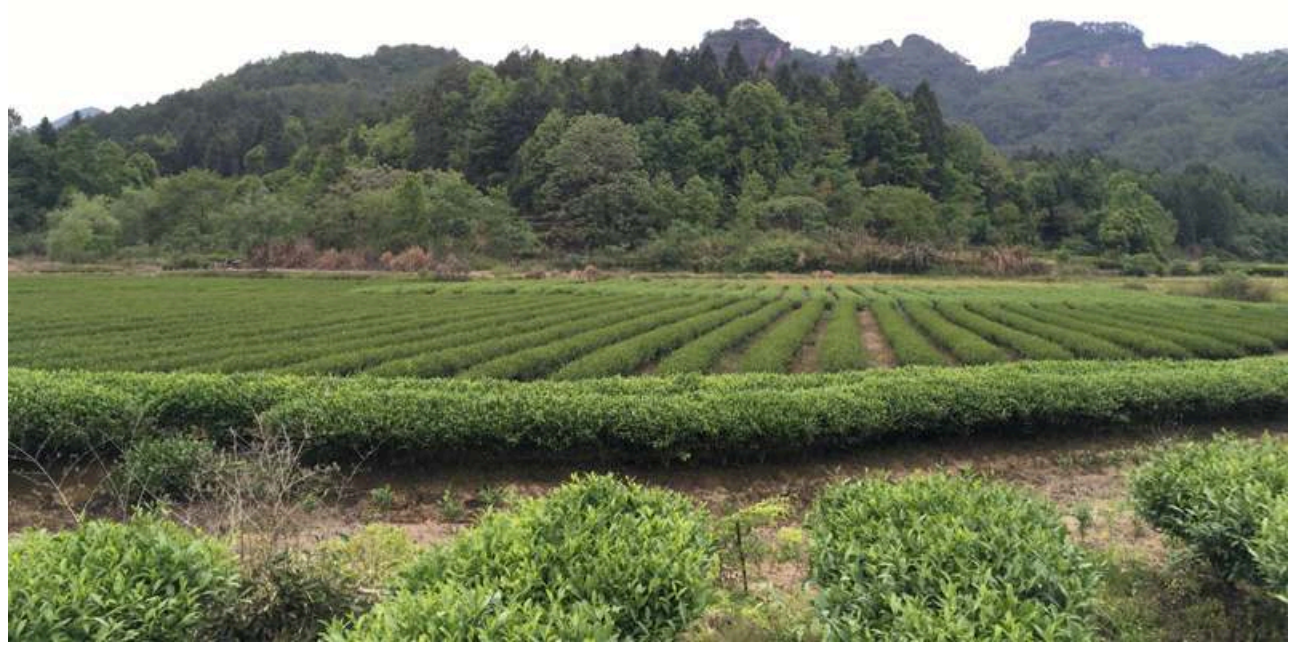

Photographiée en mai 2018. 


\section{L'image paysagère du thé, une coconstruction de l'Orient et de l'Occident?}

L'objectif est, ici, de voir comment l'image du paysage du thé s'est construite dans la littérature et la photographie entre Orient et Occident.

Selon Kubin, la littérature chinoise a commencé, à partir de la dynastie Tang, à s'intéresser à l'agriculture et aux paysans : les poètes, Wei Wang, Fu Du, Juyi Bai ont loué les espaces agricoles. En effet la vision de la « nature » et du " paysage » pendant la période des Six Dynasties était profondément influencée par les nobles qui n'ont jamais participé au travail productif si bien qu'il n'y avait ni agriculture ni agriculteurs dans l'évocation des paysages. Loin d'embellir la vie rurale, à l'image de Qian Tao (365-427), les poèmes de la dynastie Tang sont plus réalistes: Wei Wang (701-761) décrit les conditions économiques et la production agricole; et Juyi Bai (772-846), montre l'anthropisation de la nature (Kubin, 1990, p. 183-191).

Si la première mention des formes de culture du thé, qui s'apparente de fait à un récit sur les paysages du thé, remonte au VIII ${ }^{e}$ siècle, dans Le Classique du thé de Yu Lu, on peut retenir d'autres textes. Ainsi sous la dynastie Jin de l'Ouest (265-316), Yu Du écrit dans Chuanfu: «Les théiers poussent dans des endroits brumeux et humides, le sol fertile les arrose et les feuilles sont fraîches et tendres. » Et le poète Zhongyan Fan (989-1052) écrit dans son poème He Zhangmin Congshi Douchage: «Les bourgeons du théier sont prospères comme du jade, parsemés sur le théier. " Enfin, l'ouvrage de Cishu Xu (1549-1604), ChaShu, n'évoque pas seulement des plantations, mais la salle du thé, et le voyage autour du thé. Précurseur des guides touristiques, dans le chapitre du voyage, Cishu Xu mentionne la source du Rêve du tigre à Hangzhou comme une excellente eau de dégustation.

À partir de ces éléments, une typologie des descriptions du paysage dans les ouvrages traitant du thé se dessine avec :

- les plantations de théiers, leur culture et leur répartition géographique ;

- les paysages vus par le prisme de la qualité du thé. Le meilleur théier pousse toujours dans les régions montagneuses, escarpées et brumeuses; l'eau de source est la meilleure pour faire un excellent thé ;

- les meilleurs paysages pour boire le thé : soit dans la nature, soit dans le jardin classique, soit dans la salle du thé (lieu destiné à la cérémonie du thé).

19 Toutes ces descriptions plutôt techniques concernent l'environnement naturel en général et la beauté du paysage du thé en particulier y est rarement évoquée. D’une part, les élites cultivées et les "classes de loisir » urbaines qui possèdent la parole décrivent avant tout le paysage naturel (shan shui, montagne-eau) et le jardin traditionnel chinois, le paysage né de la culture et de l'activité agricole étant moins valorisé ; plus encore, la dégustation et les signes culturels attachés aux formes de consommation du thé l'emportent largement. L'acte culturel majeur est celui de la consommation si bien que les regards esthétiques sur le paysage du thé et les récits de la production et de la vie des producteurs de thé sont très peu nombreux.

De même, dans les peintures chinoises, le paysage théicole est quasiment absent ; seule sa dégustation - donc le produit fini - s'impose comme thème. Le terme " paysage » en Chine signifie «montagne-eau » 山水 ou « scène du vent et de la lumière du soleil se reflétant sur la végétation» 凤景, 凤光. Ce paysage apparait dans la peinture, sous les 
dynasties du Nord et du Sud (420-589) $)^{5}$ et les lettrés de cette époque "voient le monde avec un cœur clair et simple pour comprendre le "Tao", voyagent et apprécient le paysage à travers la peinture ${ }^{6}$. » Dans l'histoire chinoise, la représentation du paysage est ainsi limitée au paysage naturel, paysage vertical qui réunit la montagne, la rivière et la forêt, paysage qui s'oppose à la société et à la vie urbaine. Dans cette logique, les descriptions du paysage dans les ouvrages du thé tendent vers le grandiose ou le « cosmique ». En français, le terme " paysage » implique une référence à l'espace et au territoire en tant que forme appropriée et transformée par l'homme et le travail agricole. "Le paysage est à l'origine du modèle agricole, de son agriculture elle-même le produit de ce que cette société mange selon ses codes fixés par des diététiques et des rapports sociaux. » (Fumey, 2010, p. 107.) Cette conception se retrouve dans les images du paysage du thé qui apparaissent dans les dessins et photographies des Occidentaux qui parcourent la Chine à la fin de XIX ${ }^{\mathrm{e}}$ siècle.

La plupart des sources iconographiques du XIX siècle sont en effet le fait d'Occidentaux ayant voyagé ou travaillé en Chine ${ }^{7}$. Ainsi le botaniste britannique, Robert Fortune, voyage dans les années 1840 à Fuzhou et aux monts Wuyi : il évoque les terroirs et les paysages du thé ainsi que les pratiques villageoises autour de cette plante. Les illustrations de son livre Two Visits to the Tea Countries of China and the British Tea Plantations in the Himalaya, publié en 1853, donnent à voir les cultures du thé dans un paysage montagneux et marqué par les pentes (figure 10).

Figure 10. Plantation du thé

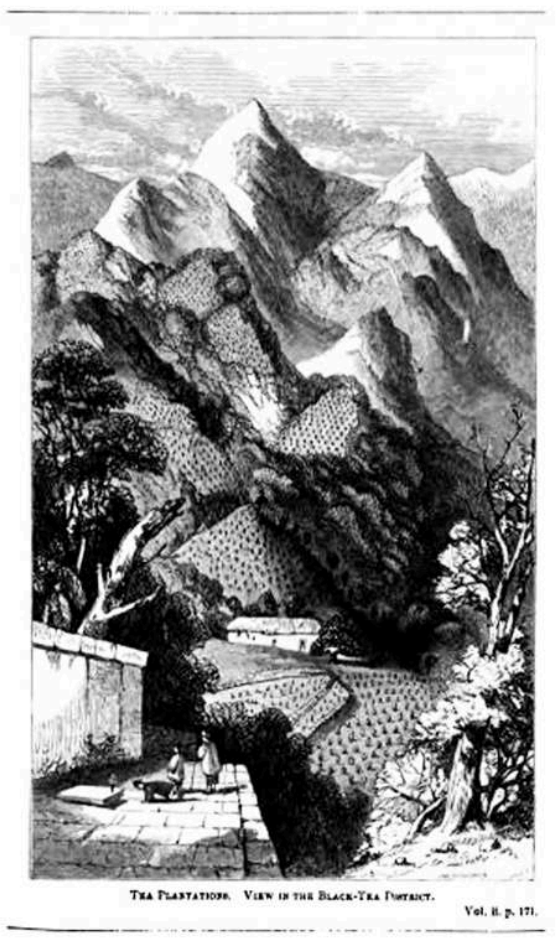

Illustration du livre de Robert Fortune, Two visits to the tea countries of China and the British Tea Plantations in the Himalaya (1853).

Source : https://www.flickr.com/photos/biodivlibrary/17641603128, Biodiversity Heritage Library. 
On peut également citer les peintures de Thomas Allom publiées China, in a Series of Views, Displaying the Scenery, Architecture, and Social Habits of That Ancient Empire, publié en 1843 (figure 11).

Figure 11. Monts Wuyi (dit aussi Wuyishan, ou Bohea Hills), province de Fujian, peinture de Thomas Allom

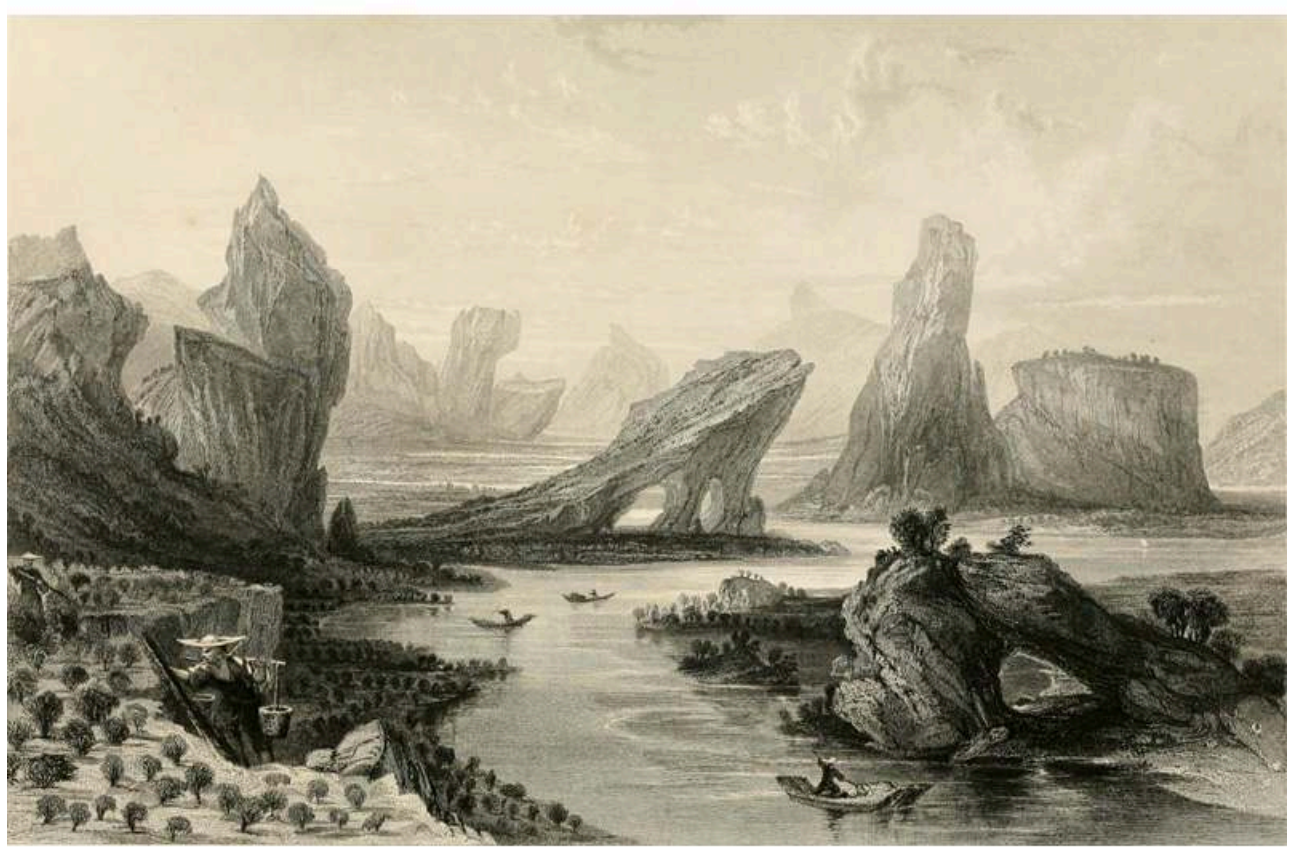

Elle a été effectuée à partir des croquis de William Alexander, qui a visité la Chine en 1793 (Allom, 1843, p. 45).

Source : https://commons.wikimedia.org/wiki/File:Woo-e-shan.jpg. 
Figures 12. The Tea Plant
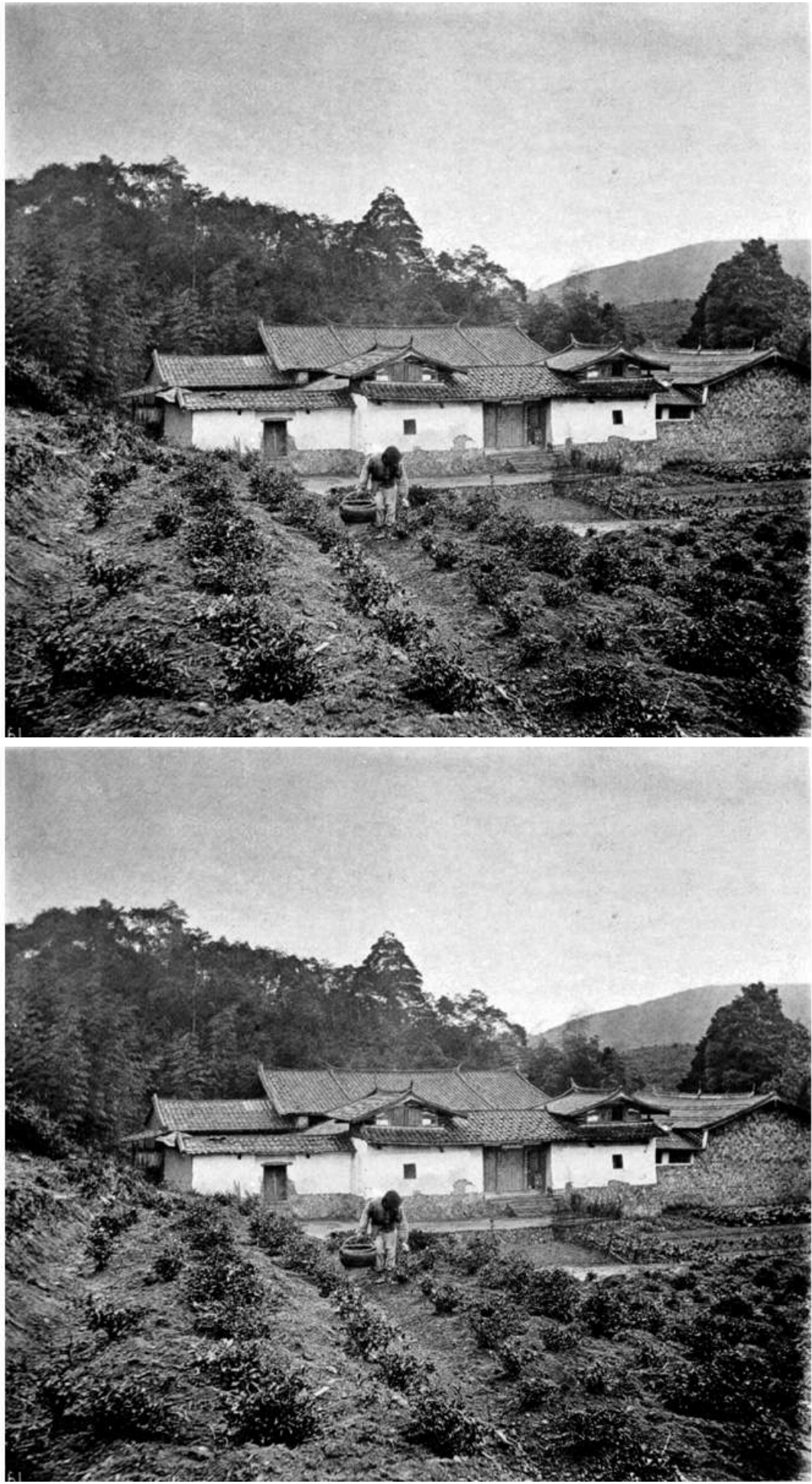


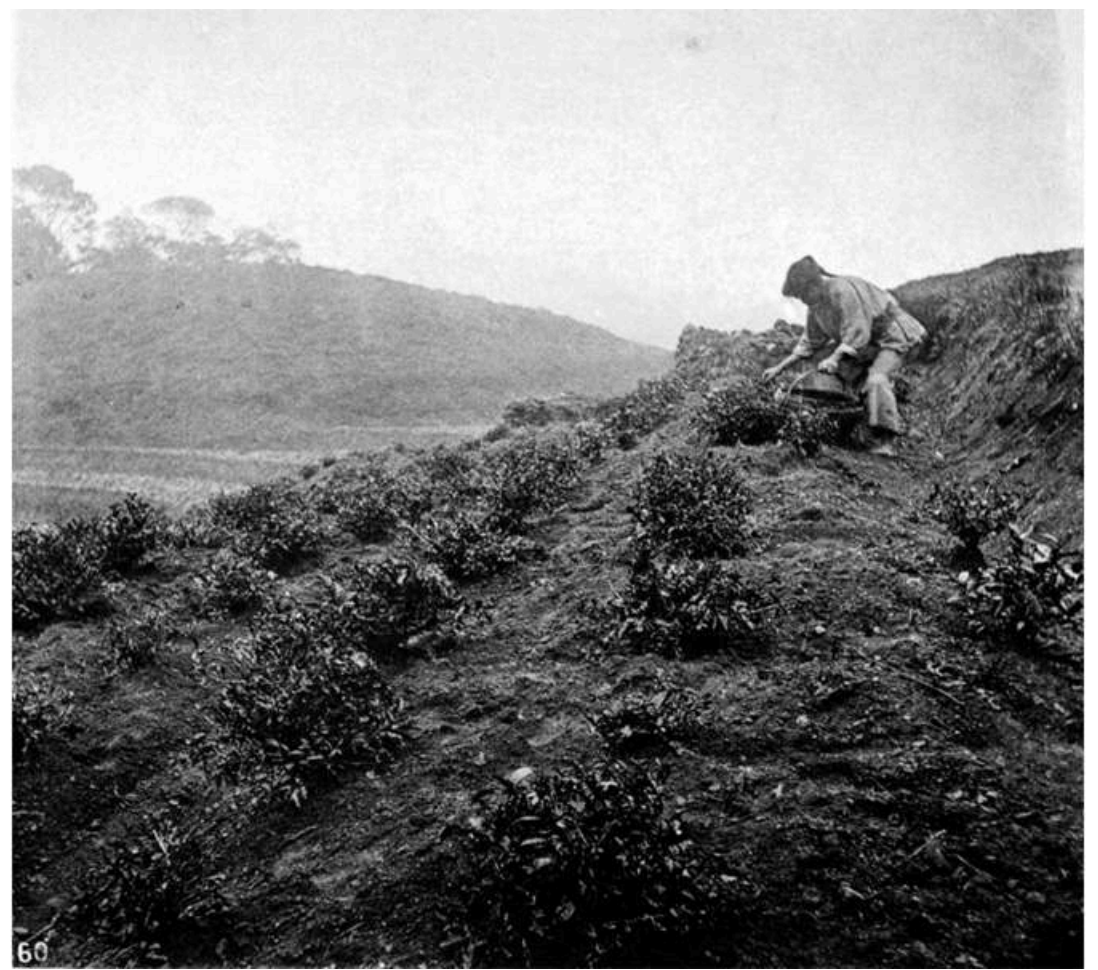

Photographié par John Thomson avant 1898.

Sources:

https://commons.wikimedia.org/wiki/File:THE_TEA_PLANT_1.jpg https://commons.wikimedia.org/wiki/File:THE_TEA_PLANT_2.jpg

Quant à l'explorateur écossais John Thomson, un des premiers photographes à parcourir l'Extrême-Orient, il a immortalisé les coutumes et la vie du XIX siècle en Asie. Voyageant dans la province de Fujian dans les années 1870, il a publié de nombreuses photos de plantations de thé (figure 12).

24 La quasi-absence d'images sur le paysage du thé réalisées par les Chinois avant le XX siècle s'explique tant du fait de la guerre et du retard économique et technologique de la Chine d'alors que d'une autre conception du paysage. Les documents disponibles faits par les Occidentaux illustrent leur point de vue : c'est une vision occidentale, montrant une curiosité pour l'Orient, parfois condescendante. Ces données permettent toutefois, en les recoupant, de reconstituer les paysages du thé de cette époque. En comparant avec les ouvrages chinois antérieurs, on constate un certain nombre de constantes. Ainsi les jardins du thé en Fujian présentent au XIX ${ }^{\mathrm{e}}$ siècle une double morphologie, avec des formes régulières et irrégulières. Ils sont tantôt sur les pentes de haute montagne, tantôt sur les versants de colline mais aussi dans les plaines. Dans les plaines et sur les pentes douces, ils s'épanouissent sur des superficies importantes alors qu'à proximité des villages, des potagers, au pied de montagnes, ou sur les rochers abrupts et escarpés, ils sont cultivés sur de petites parcelles de quelques unités parfois.

Cette mise en regard de corpus variés tant chinois qu'occidentaux dans une approche diachronique souligne les formes de l'émergence du paysage théicole. Le processus de domestication du théier a conduit à un paysage régulier, ordonné qui porte encore certaines marques du passé (grands arbres, ancrage forestier). Si les images du paysage du thé sont présentes très tôt dans la peinture et la littérature chinoises, elles restent tributaires d'une approche culturelle du thé où la dégustation et la consommation sont 
les actes nobles par excellence. De nouvelles images vont apparaître avec l'arrivée des Occidentaux, notamment par la photographie. Il faut lire l'invention du paysage du thé dans la rencontre de ces deux approches. Jusqu'à la fin du XIX ${ }^{\mathrm{e}}$ siècle, l'accès aux écrits et aux images reste limité en Chine alors qu'on entre, au $\mathrm{XX}^{\mathrm{e}}$ siècle, dans une phase majeure de démocratisation, d'accès à l'image: celle-ci devient alors un outil commercial et communicationnel.

\section{De la marginalité à la banalisation du paysage du thé dans la publicité}

La place du paysage du thé dans les images publicitaires s'est accrue au fil du temps: d'abord absent, puis marginal, il est devenu aujourd'hui banal. Avec la photographie et d'autres médias, les consommateurs ne sont plus seulement les élites mais aussi des catégories plus populaires qui ont leurs propres représentations et un imaginaire différent sur le thé et ses images.

\section{Un paysage en creux}

À la fin du XIX et au début du XX ${ }^{e}$ siècle, la plupart des photographies reflétant la vie et les coutumes locales de la Chine ont été prises par des Occidentaux. L'utilisation de la technologie photographique par les Chinois n'a vraiment commencé que pendant le mouvement d'auto-renforcement ${ }^{8}$ et l'objectif était la réalisation de portraits, dans un but commercial. Les photographies de paysage faites par des Occidentaux seront toutefois associées à la peinture chinoise dans nombre de publicités commerciales 9 . Ainsi, dans les années 1920-1930, la publicité utilise des photographies qui s'inspirent du style, de la perspective ou des peintures à l'huile occidentales. Mais afin de siniser la publicité, et de garantir aux yeux des consommateurs l'authenticité des produits, leurs auteurs y intègrent des pratiques culturelles très codifiées, telles que la poésie, la peinture et la calligraphie.

Les images publicitaires (figures 13, 14 et 15) montrent ainsi des scènes de dégustation associant des calligraphies. Dans les années 1930, le texte calligraphié est très présent dans les publicités avec des textes en police artistique, parfois avec quelques motifs. C'est bien souvent un simple pot de thé carré géométrique qui représente le thé accompagné d'un texte qui vante les vertus médicinales du breuvage et cite la marque du producteur. Ces textes ne sont pas seulement des explications complémentaires aux images; leur rôle est d'intégrer des symboles culturels traditionnels aux publicités par un choix de motifs et de typographies archaïsants. 
Figure 13. Emballage de thé des années 1920-1940

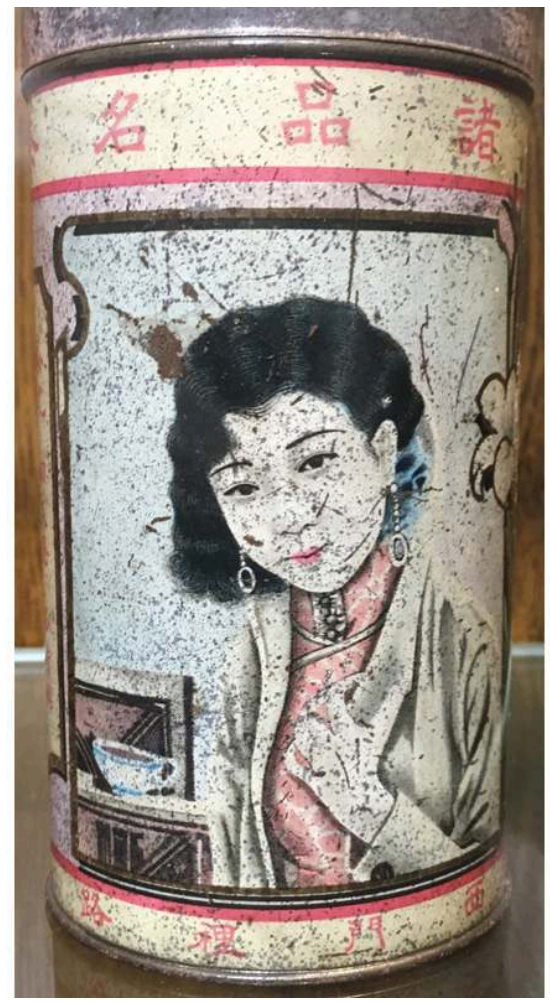

On trouve de nombreux portraits, souvent des actrices/chanteuses de l'époque, en train de prendre le thé dans une maison ou un jardin chic avec un service à thé anglais. Photographié en mai 2018 à l'entreprise Xiang JiangMingYuan. 
Figure 14. Emballage de thé des années 1920-1940

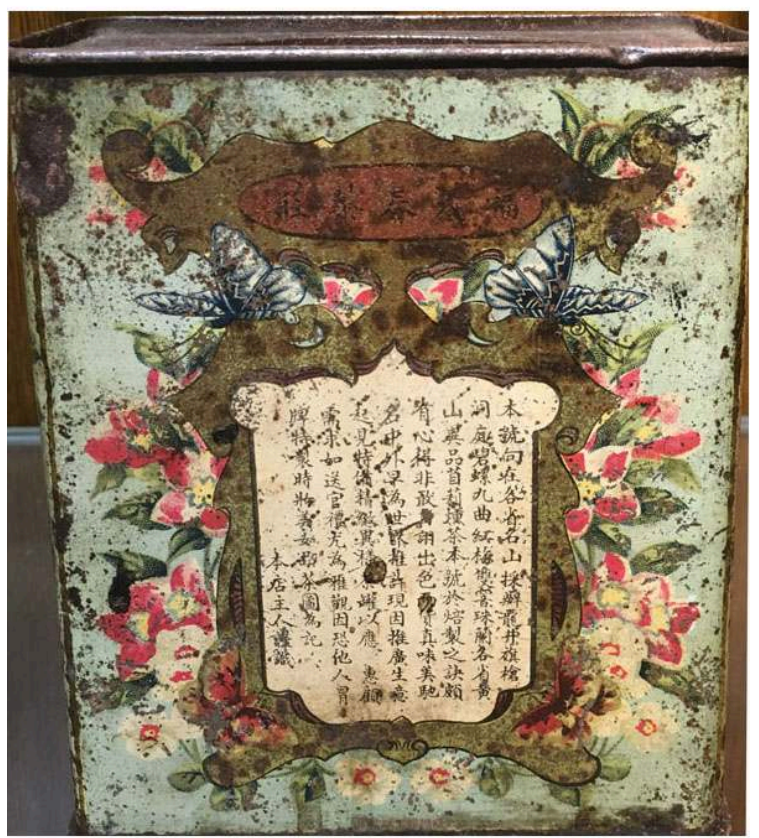

La figure représente la photographie d'une boîte de thé métallique avec des couleurs lumineuses et des détails complexes ; le contenu textuel détaille les variétés de thé vendues par le producteur. Le texte justifie aussi la présence d'une photographie de femme sur le devant de la boîte : comme la photographie est moins facile à imiter qu'une calligraphie, il s'agit d'éviter la contrefaçon.

Photographié en mai 2018 à l'entreprise XiangJiangMingYuan 
Figure 15. Publicité du thé en Fujian

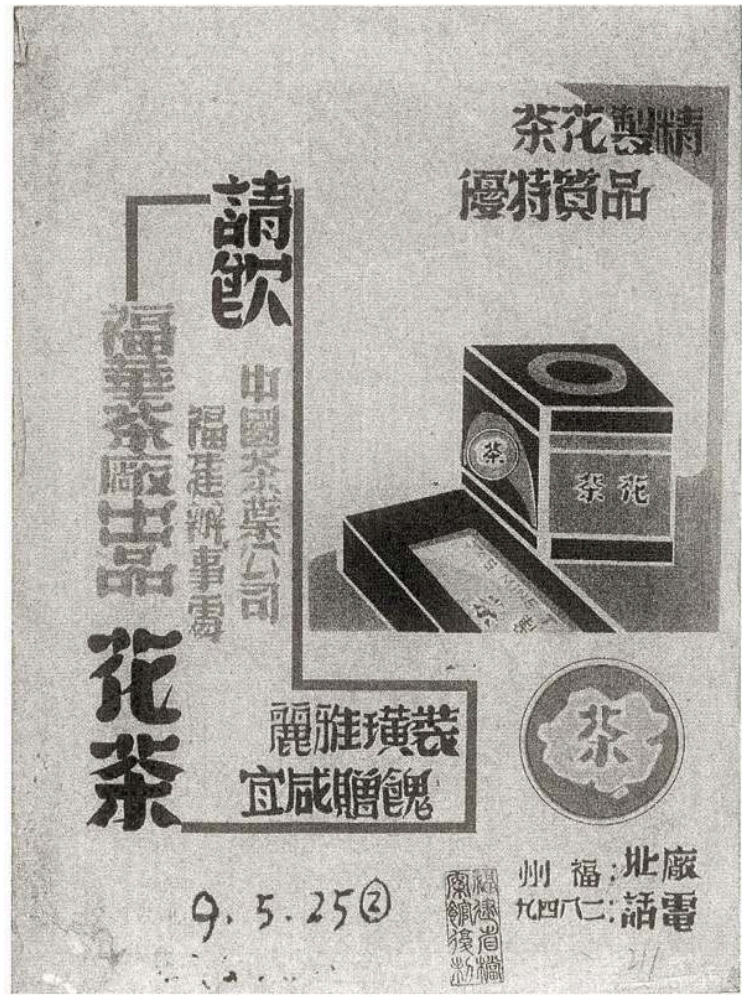

Magazine Min Cha Ji kan, vol. I, nº 3, 1925 : cette image est une publicité issue d'un magazine professionnel sur le thé : le dessin évoque une perspective, les motifs et les couleurs sont plus discrets sans doute car les consommateurs ciblés sont des catégories socioprofessionnelles favorisées et des connaisseurs du secteur du thé

Dans les années 1920-1940, les portraits caractérisent la plupart des images publicitaires du thé (figures 16 et 17). A contrario la présence de paysages théicoles ou d'autres éléments paysagers est relativement rare dans les publicités de cette période et, quand ils existent, ils apparaissent toujours en arrière-plan : sous la forme d'un jardin traditionnel chinois ou un jardin du thé. La plupart du temps, ce sont des jardins ou des paysages intérieurs (figures 18 et 19). 
Figures 16. Emballages de thé des années 1920-1940

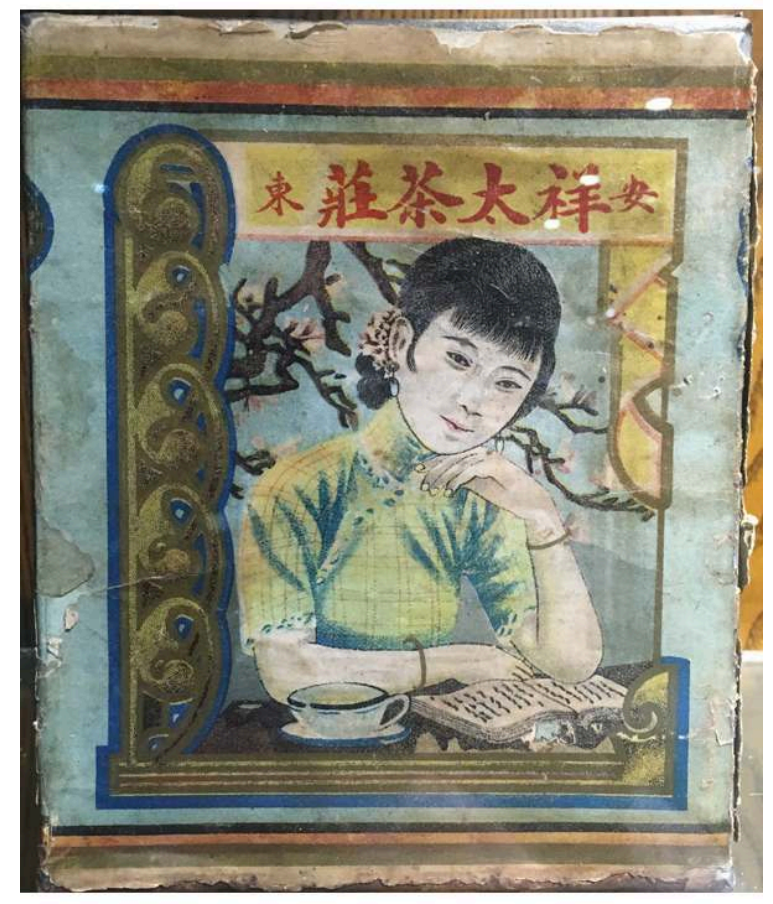

Sur ces deux figures, des femmes bien habillées portent des bijoux, l'une lit avec une tasse de thé, l'autre boit dans un jardin fleuri. Photographiés en mai 2018 à l'entreprise Xiang JiangMingYuan. 
Figure 17. Emballages de thé des années 1920-1940.

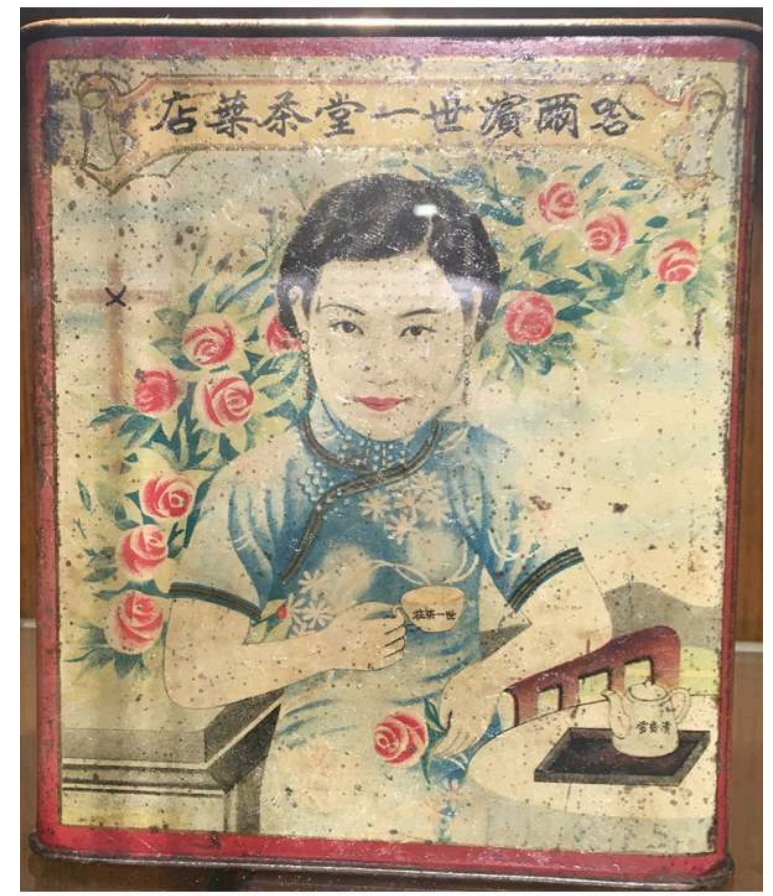

Sur ces deux figures, des femmes bien habillées portent des bijoux, l'une lit avec une tasse de thé l'autre boit dans un jardin fleuri. Photographiés en mai 2018 à l'entreprise Xiang JiangMingYuan. 
Figure 18. Emballage de thé des années 1920-1940.

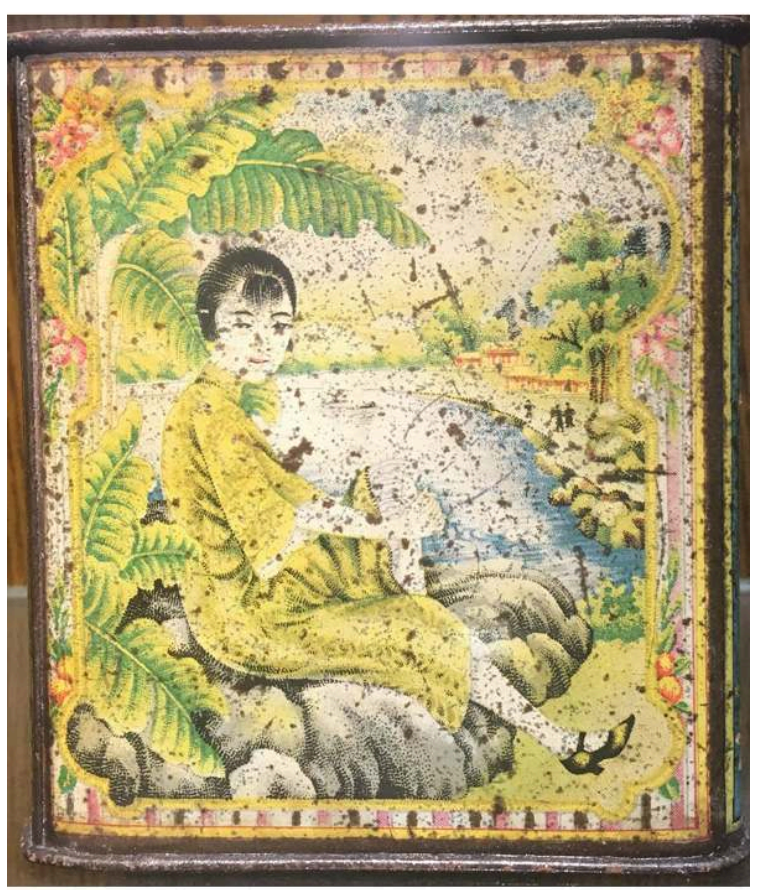

La femme est assise sur une rocaille dans un jardin traditionnel chinois, sourit légèrement en tournant sa tête vers le spectateur : son petit pied droit est légèrement retroussé de manière provocante, le gauche est caché sous la jupe. Photographié en mai 2018 à l'entreprise XiangJiangMingYuan. 


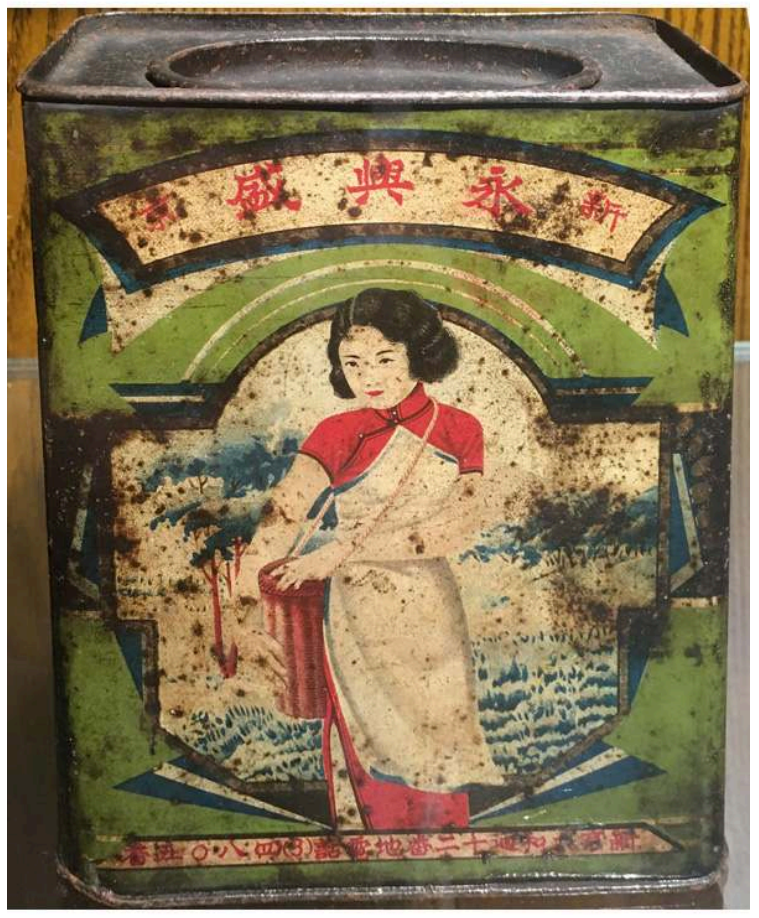

Ce portrait représente une scène de récolte du thé avec une femme en gros plan alors que beaucoup de théiculteurs sont des hommes. Cette dernière est habillée en Qipao (vêtement féminin traditionnel chinois, à la mode à Shanghai au début du XXe siècle parmi les femmes bourgeoises urbaines), ce qui peut sembler absurde avec, au fond, quelques éléments de paysage du thé. Photographié en mai 2018 à l'entreprise XiangJiangMingYuan.

Dans les rares publicités illustrées par des paysages et des théiculteurs, le paysage du thé est parfois remplacé par un paysage de rizière ou un champ de blé - ce qui n'est pas sans étonner ${ }^{10}$. Ces substitutions illustrent une ignorance de la culture du thé, une méconnaissance de ses paysages agricoles tant par les commerçants en ville que par les consommateurs urbains ${ }^{11}$.

Wendy Joy Darby, dans son livre Landscape and identity, souligne que le paysage est un champ dominé par les hommes, «un appendice de l'identité, [qui] tout comme les épouses, les amants et les filles étaient des appendices aux hommes qui possédaient la terre et géraient le pays" (Darby, 2000, p. 2). Il en est ainsi du thé. Les images publicitaires largement féminines, le choix même du paysage particulier dans lequel elles sont placées reflètent clairement une société patriarcale dans laquelle le désir, à tout le moins le regard appuyé des hommes, est imposé aux femmes, ces dernières acceptant cette place ou rêvant de la vie imaginée de la femme illustrée. En outre, la présence d'éléments figurant l'urbain, de vêtements chic et d'objets occidentaux dans les publicités témoigne de la manière dont les négociants en thé imaginent leur clientèle, et leur préférence : des consommateurs aspirant à un mode de vie urbain et moderne et imitant la vie culturelle occidentale. 
Figure 20. Emballage de thé des années 1920-1940

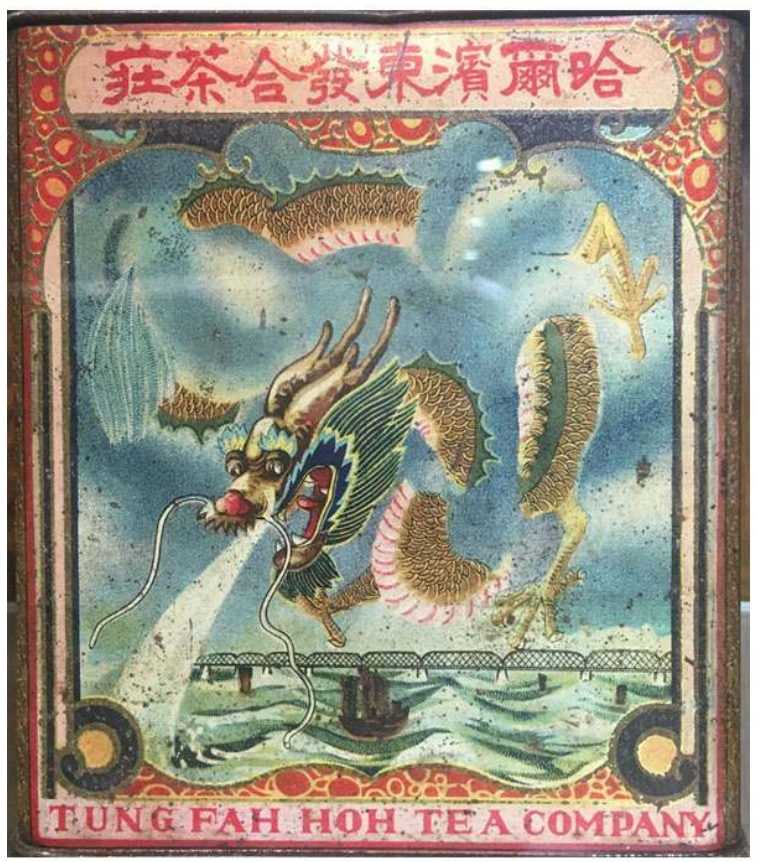

Les commerçants sélectionnent parfois pour leurs publicités une personne âgée, des animaux comme la grue ou le dragon, tous symboles de la longévité et des vertus médicinales attribuées au thé. Photographié en mai 2018 à l'entreprise Xiang JiangMingYuan. 


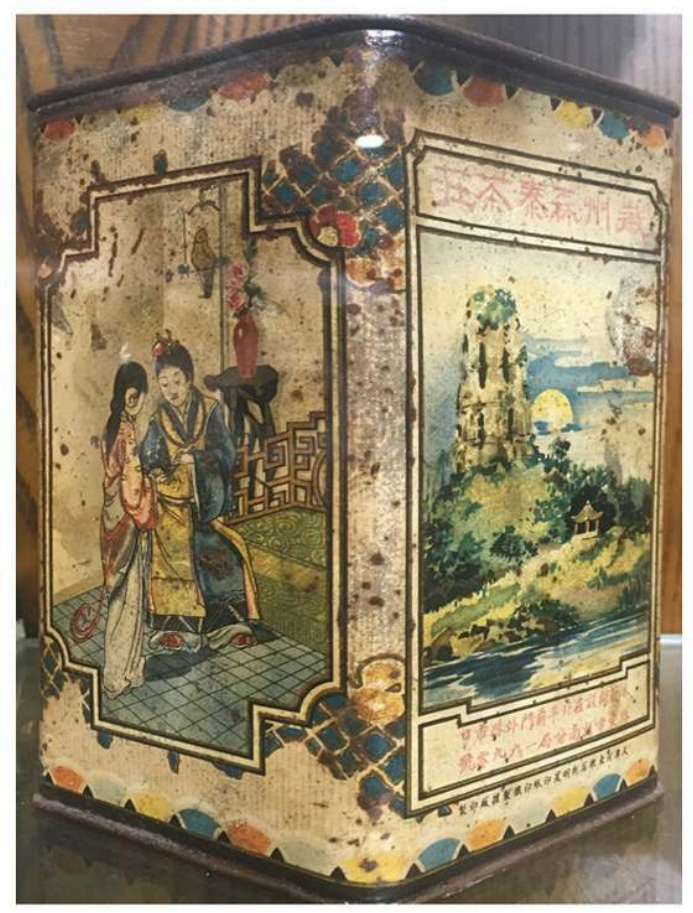

Ce paysage met en scène très nettement des montagnes, l'eau, le soleil couchant et l'herbe ; on ne peut toutefois affirmer s'il y a un jardin de thé ou non, mais les arbres au fond sont peut-être des théiers. Photographié en mai 2018 à l'entreprise XiangJiangMingYuan.

Le paysage du thé n'est pas complètement absent des images publicitaires des années 1920-1940, mais il est étrangement discret, en creux. La calligraphie, les personnages sont toujours plus grands, plus importants et remplissent le format au point de l'écraser quand il n'est pas tout simplement remplacé par un paysage imaginaire et indéterminé (figures 20 et 21).

\section{Les icônes paysagères}

Après la victoire de Mao, le pays entre dans l'ère de l'économie planifiée et le thé devient un monopole d'État. Sans entreprises privées, avec une prédominance des combats idéologiques sur les questions économiques, la publicité disparaît, remplacée par la propagande, d'où la difficulté de trouver des images du thé dans les archives.

La publicité renaît après les réformes et l'ouverture en 1978 avec l'utilisation d'images nouvelles. En même temps, le tourisme se développe à partir des années 1980, le citadin quitte sa ville le temps d'un week-end pour parcourir montagne et campagne. Et la publicité s'adapte: des icônes paysagères du thé s'imposent alors d'autant que le paysage a une fonction cognitive ; "La photographie va lier le produit au paysage. » (Fumey, 2010, p. 107.) Ainsi, de nombreux emballages sont aujourd'hui illustrés d'images paysagères, y compris le thé. L'icône paysagère thé est un symbole de la nature, du terroir et une garantie de la sophistication et de la qualité des produits.

La figure 22 représente le premier design de thé Dahongpao commercialisé en 1985 après la réforme et l'ouverture (figure 22) : une feuille de thé rouge a été choisie pour 
symboliser cette espèce de thé, Dahongpao signifiant grande robe rouge. Plus généralement, les paysages retenus dans la publicité du thé n'évoquent plus seulement le paysage de montagne et de rivière ; surtout ils jouent sur les échelles, entre grand paysage et parcelle de proximité, généralement sous forme de photographies (figure 23). Panneaux d'affichage, brochures, magazines et pages Internet, etc. en sont autant d'exemples.

Figure 22. Premier design de thé Dahongpao commercialisé en 1985

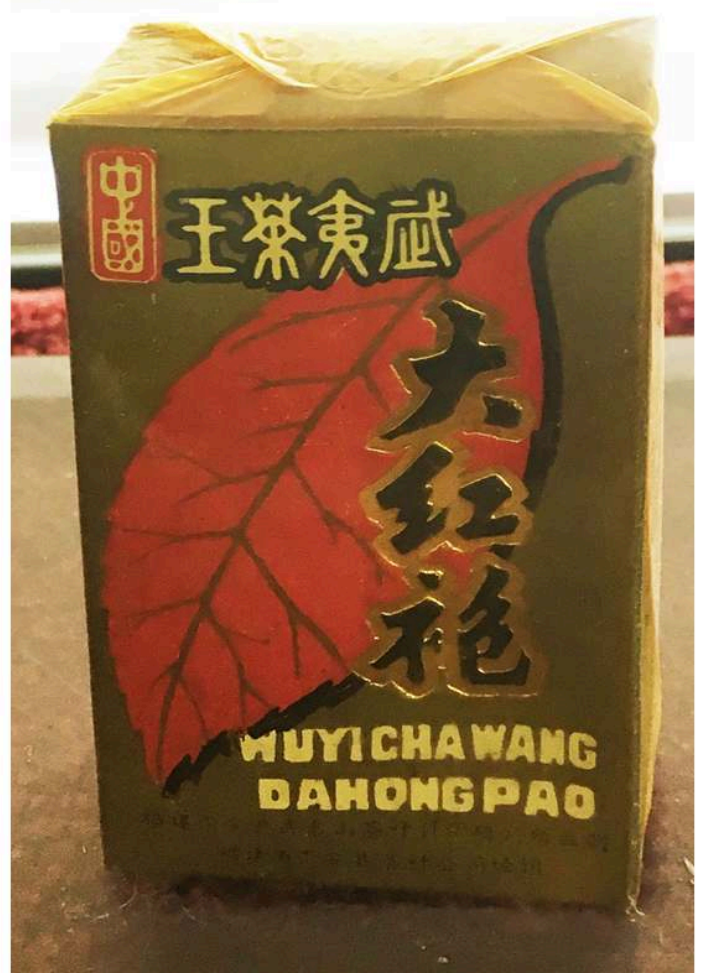

Photographié en mai 2018. 


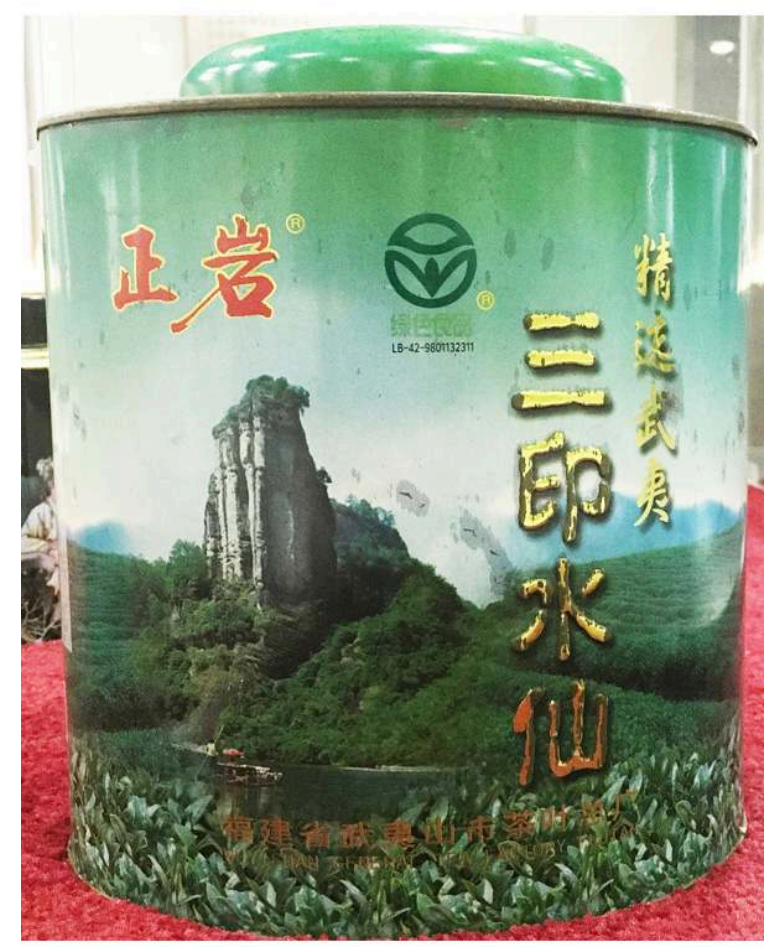

Photographié en mai 2018.

En 1995, la librairie Life, Reading and Knowledge, « ressuscite » Triple Life Weekly à Pékin et, depuis 2013, Triple Life Weekly publie chaque année des numéros hors série sur le thé : ainsi Le Thé vert, Kong Fu Cha, Nourriture et Alcool chinois, Thé et Vin, Le Secret du bon thé, etc. Les photos du paysage théicole de Fujian y sont particulièrement nombreuses. Attachées à montrer la « vraie » vie des producteurs de thé et le " vrai » paysage du thé, elles mettent l'accent sur les caractéristiques géographiques du thé et le travail des paysans dans les plantations.

\section{Artialisation du paysage}

Judith Williamson indique dans son livre Decoding Advertisements que dans la publicité moderne, il y a de moins en moins de supports physiques traditionnels, la tendance étant de convertir les publicités de produits en symboles (Williamson, 1978, p. 17-34).

Aux monts Wuyi, deux quartiers sont célèbres pour leurs boutiques d'emballage qui occupent pratiquement toutes les rues. À l'exception des grandes chaînes de magasins de marque, les détaillants et les grossistes se fournissent en emballages dans ces quartiers. On y trouve toutes sortes d'emballages (en sachet, en boîte métal, en paquet, etc.) et les designers présents font des dessins sur-mesure à la demande! Nombre de boutiques se sont d'ailleurs transformées en véritables agences de packaging au service des commerçants. Les emballages contemporains sont beaucoup plus variés avec une gamme importante de dessins, et ils indiquent clairement le nom des fabricants et l'origine de production. Aux photographies de paysages s'ajoutent des motifs plus géométriques, des figures paysagères artialisées, des couleurs éclatantes, des formes diversifiées (figure 24). Les images paysagères sont principalement des photographies 
en couleurs qui évoquent le relief et la morphologie du jardin ou la forme des feuilles ou des théiers, et des dessins abstraits inspirés des éléments paysagers (figure 25, 26). Le jardin du thé sous les nuages, sur des collines et des montagnes qui se chevauchent vient ainsi révéler le terroir et le climat propres au thé. La forme du théier et de la feuille de thé reflète également sa variété distinctive.

Figure 24. Boîtes de thé dans une boutique d'emballage sur mesure aux monts Wuyi.

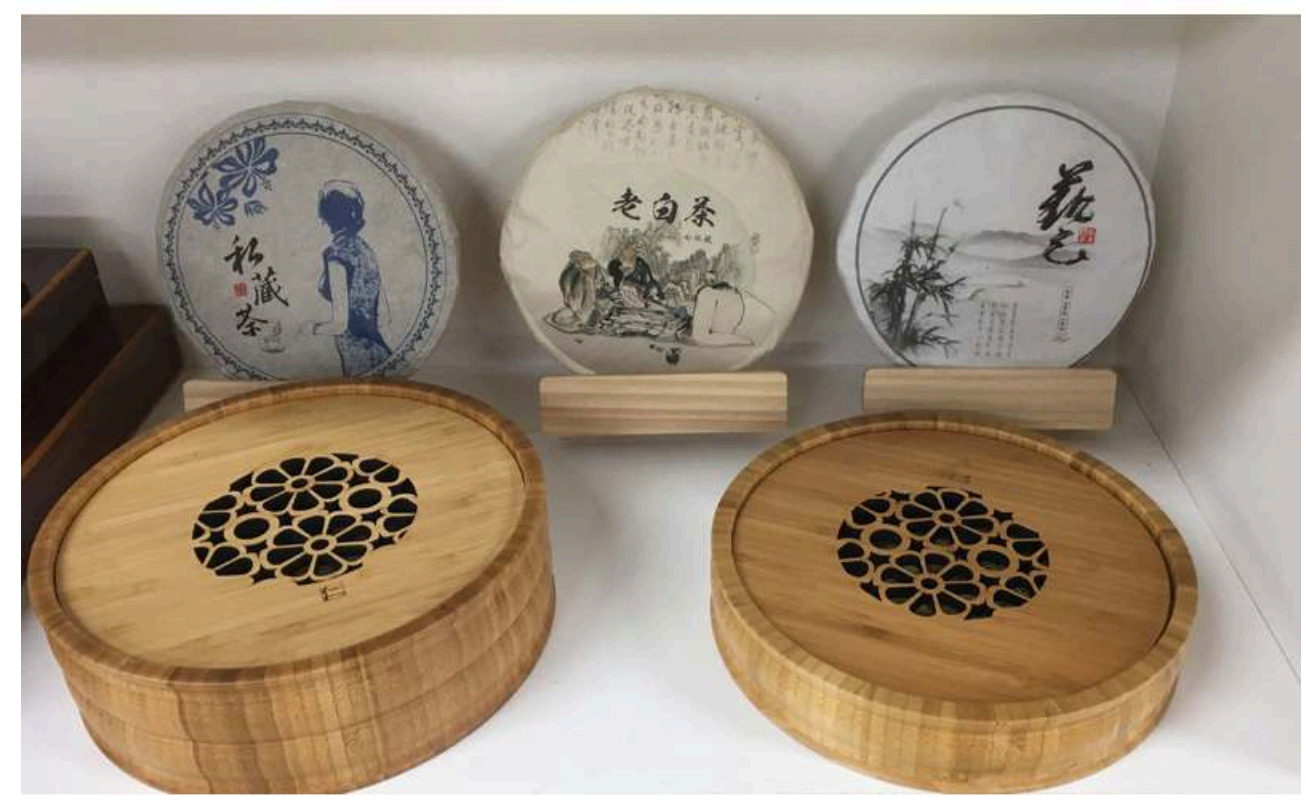

Photographiées en avril 2018 
Figure 25. Emballage du thé « Chunlun » avec paysage du thé

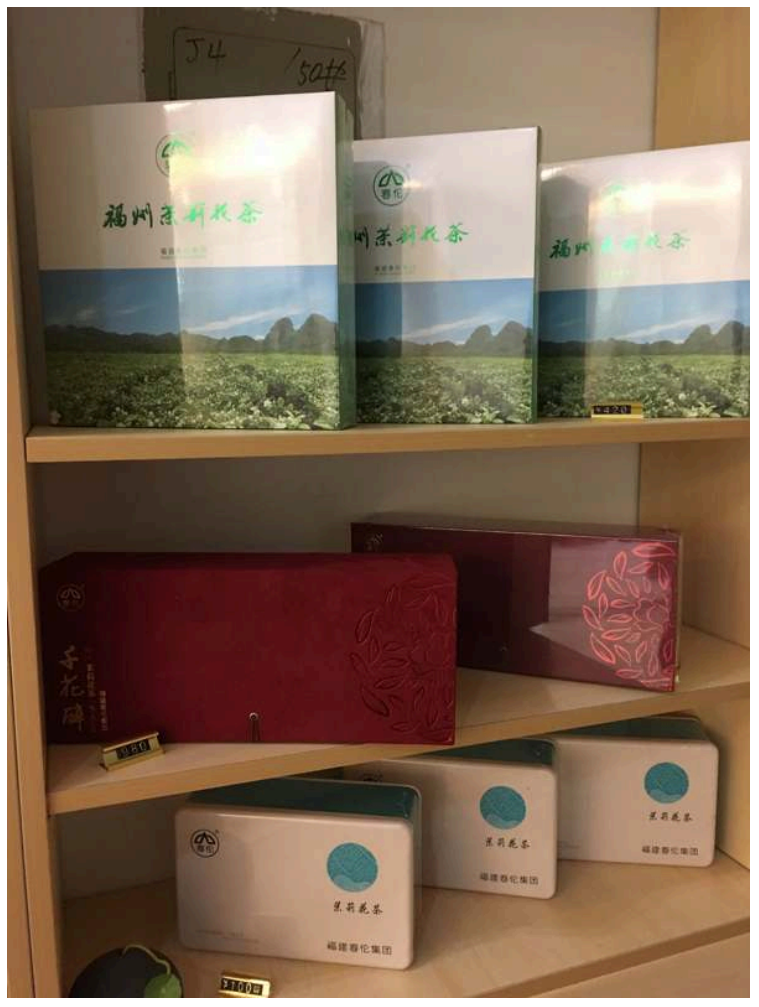

Photographié en mai 2018.

Figure 26. Emballage du thé «Chunlun » avec paysage du thé

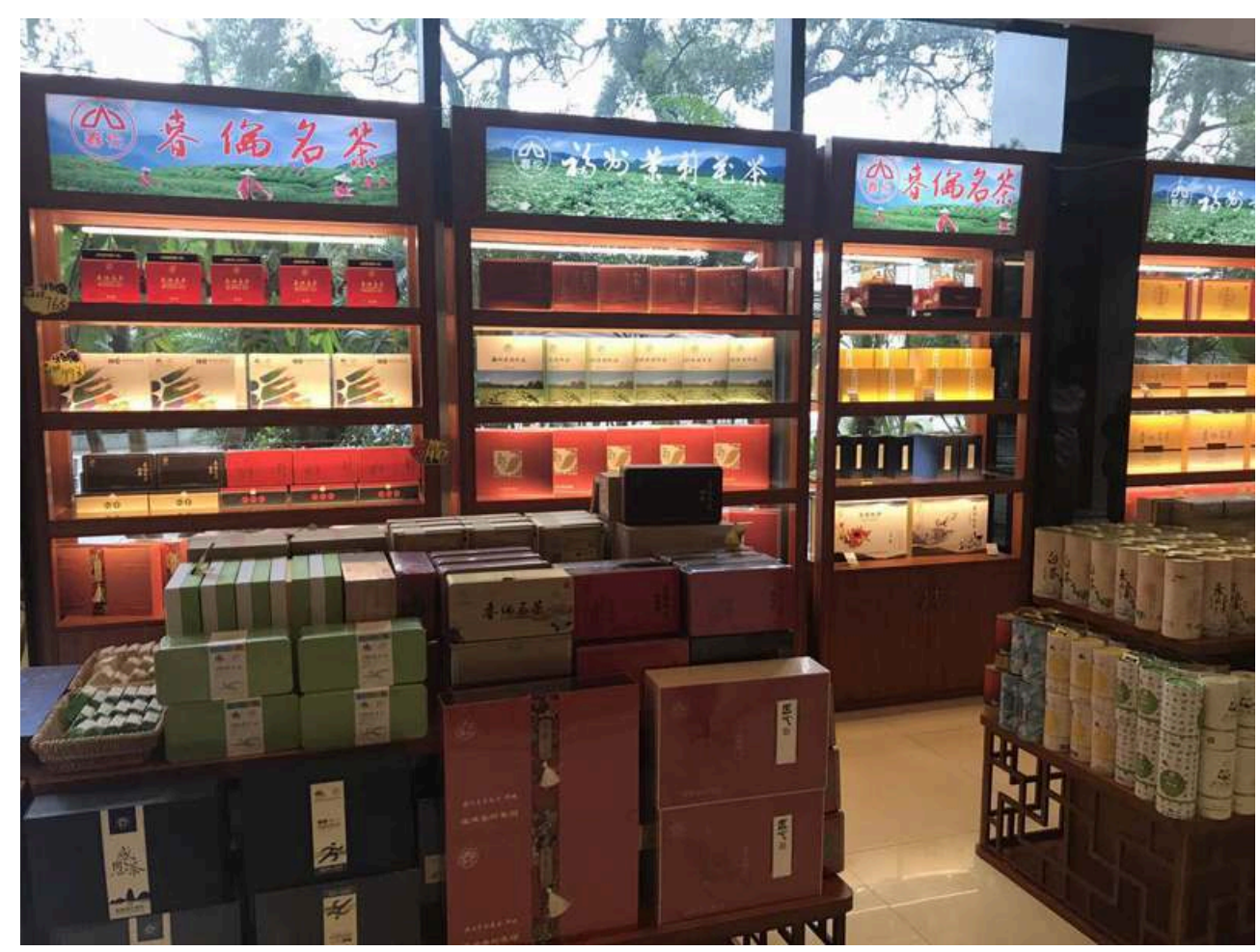

Photographié par Chunlun Groupe. 
On mesure les évolutions de l'image du thé depuis plus d'un siècle avec l'urbanisation de la Chine et la montée d'une classe moyenne. Les références ou imageries sur le thé et son paysage diffèrent selon que l'on soit lettré urbain et consommateur de thé, ou agriculteur. Les premiers perçoivent le thé comme un symbole du tao et du zen qui pousse naturellement entre montagnes et rivières : c'est un paysage où l'homme, le paysan besogneux et son travail des champs sont cachés et remplacés par la citadine chic à laquelle le consommateur des villes peut s'identifier. En revanche, pour les agriculteurs, le paysage théicole signifie leur famille, leur mode de vie, la terre qu'ils exploitent: c'est la trace laissée par leurs ancêtres, c'est l'héritage des traditions historiques et c'est le livre qu'ils continuent à écrire.

\section{Touristification et compression du paysage}

40 La culture du thé remonte sans doute à la dynastie Xia (2070-1600 av. J.-C.) avec le souverain civilisateur de la Chine, Yandi (aussi appelé Shennong). Le thé n'a jamais quitté l'Extrême-Orient jusqu'aux grandes découvertes. Il a ensuite voyagé vers l'Europe, où il s'est confronté à un système culturel exotique, et y a développé un langage complètement différent, à l'image du café, du vin, et de la tequila, boissons excitantes devenues des médiateurs universels, « utilisées par des civilisations entières pour signifier le désir », qui non seulement ont façonné la culture locale, mais aussi ont «[fouillé] la chair pour exprimer [leurs] pouvoirs » en créant un codage international commun (Onfray, 1995, p. 131).

\section{Touristification du paysage}

41 Avec le développement du tourisme dans les années 1980, du marketing et de la publicité, les plantations de thé et le paysage du thé attirent de plus en plus de visiteurs. Bien que certains lettrés chinois aient, historiquement, voyagé pour déguster le thé, l'ère du tourisme de masse a débuté dans les années 1980 et 1990 en Fujian.

Nombre d'entreprises de thé se convertissent au tourisme en imitant le modèle d'œnotourisme : on voit fleurir les «châteaux » du thé, les routes du thé ; on parle de terroir à thé (le terme chinois est « champ de montagne»), etc. L'office du tourisme des monts Wuyi diffuse ainsi des informations sur la route du thé dans des brochures touristiques et sur son site Internet: ce sont notamment des cartes et des circuits recommandés. La notion de "route du thé » est ancienne mais historiquement il s'agissait de la route commerciale suivie par les caravanes vers la Mongolie et la Russie alors que les routes actuelles sont beaucoup plus courtes, limitées à une région.

L'exemple du complexe touristique de l'entreprise XiangJiangMingYuan, ajouté en 2015 à une fabrique de thé des monts Wuyi, est significatif. Ce parc industrialo-touristique couvre une superficie de 170 hectares, comporte une architecture en béton imitant le style traditionnel chinois en bois, un jardin traditionnel, un pavillon et un corridor de style chinois entre les zones de visite (figure 27). Le centre du parc est occupé par un lac artificiel et, sur ses rives, différents théiers ont été plantés. On trouve aussi un musée de la culture du thé, un autre sur l'histoire de l'entreprise, un atelier de fabrication du thé à la main et son pendant industriel, des salles où les produits sont exposés ainsi qu'un centre de dégustation. Le site de l'usine se situe au milieu d'une zone de collines, naturelles, couvertes de théier. Le musée présente une maquette 
topographique des zones de culture du thé des monts Wuyi, et leur histoire, les différentes espèces de théiers, le processus de fabrication, les outils, les coutumes des thés des monts Wuyi. Dans l'atelier manuel, les artisans simulent et montrent le processus de fabrication du thé. Et dans l'atelier industriel, on peut voir le processus de production industrielle. Dans le centre de dégustation, trône une immense théière de 10 mètres de haut. Ce lieu est une salle de spectacle où sont organisées régulièrement des cérémonies du thé et des concours de thé, avec des acteurs en vêtement archaïsant qui utilisent des objets en style antique. Un centre commercial est installé juste à côté, où les touristes peuvent déambuler et faire des achats après le spectacle.

Figure 27. Carte de visite de l'usine XiangJiangMingYuan

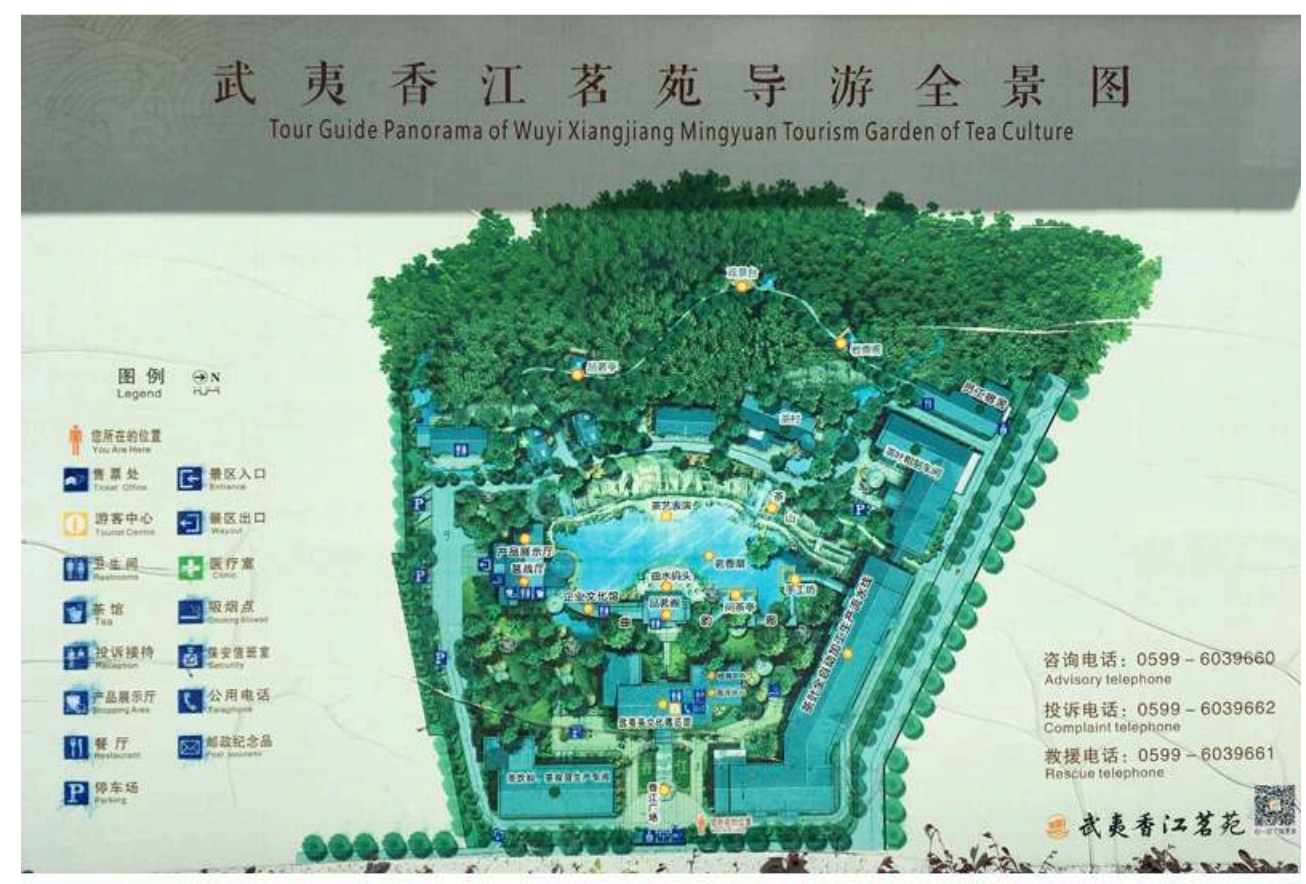




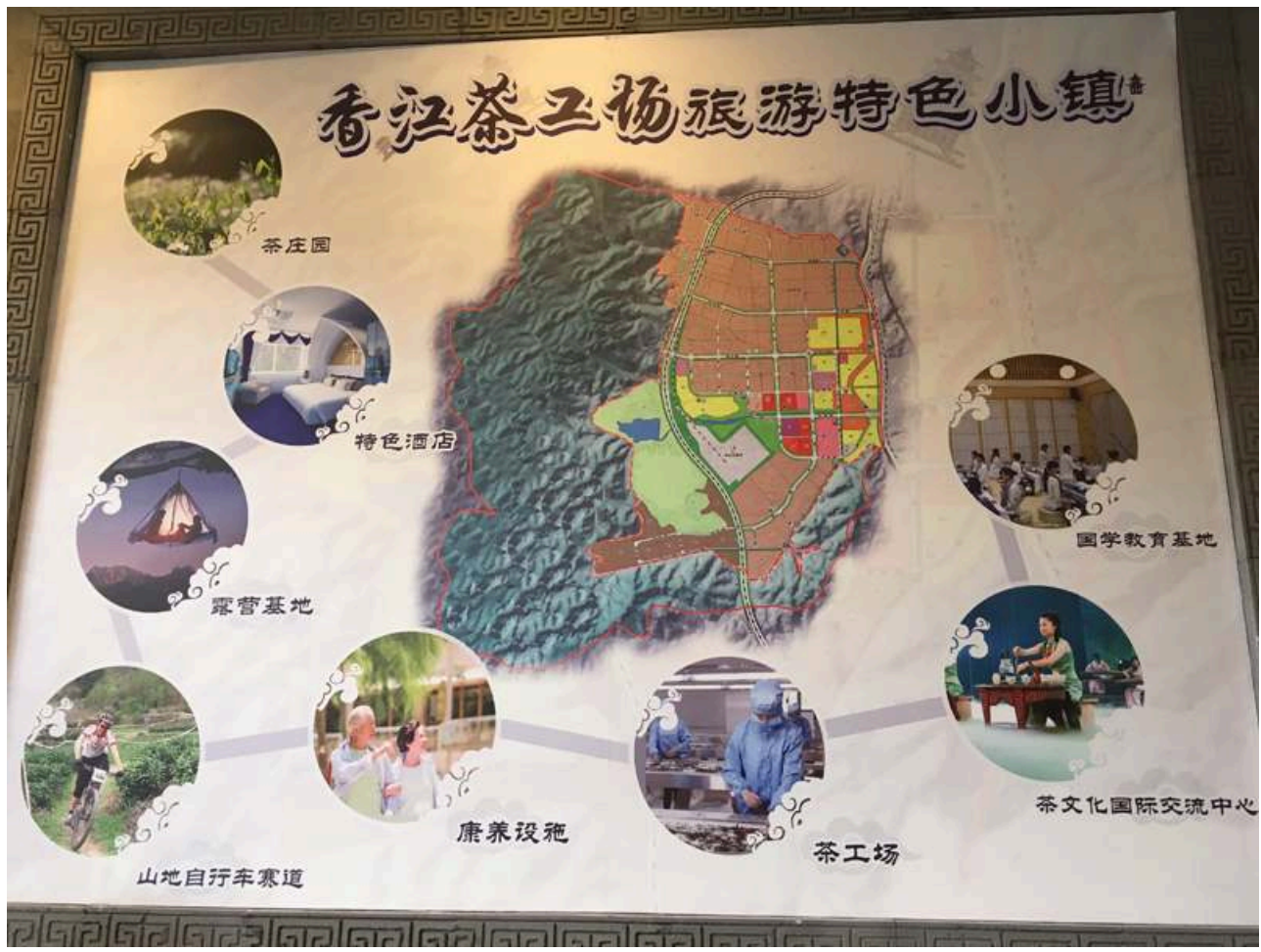

Avec les mentions « château » de thé et « village caractéristique ».

En outre, ces installations touristiques comprennent un « château » du thé, un hôtel sur le thème du thé, un camping, des pistes de VTT, un centre de santé, un centre d'échanges internationaux et le centre pédagogique du confucianisme (figure 28). C'est l'un des parcs touristiques les plus complets et les plus astucieusement réalisés que j'ai pu voir en Chine : l'entreprise y a fortement investi et a mené des enquêtes sur tous les aspects autour $\mathrm{du}$ thé. Mais la réalisation procède aussi d'une sorte de Disneylandisation autour du thé avec des constructions artificielles. Sur la carte de l'usine, le «château » de thé est localisé, en se référant aux châteaux bordelais et au "village caractéristique ${ }^{12}$ ». XiangjiangMingYuan utilise donc des modèles étrangers pour attirer les touristes en leur proposant une expérience totale, sans rencontrer un franc succès, hormis quelques touristes chinois âgés.

Dans ce tourisme autour du thé, l'image du thé et de son paysage a été affaiblie; le château, l'hôtel, le camping, le jardin chinois, l'architecture traditionnelle s'apparentent à des « objets tocards » dans un « univers du pseudo-environnement, du pseudo-objet, (qui) fait les délices de tous les "créateurs" "fonctionnels" » (Baudrillard, 1996, p. 165). Plus encore, le "vrai » paysage du thé de terroir, investi par un paysage méticuleusement reconstruit, devient un pseudo-paysage culturel. Les touristes locaux chinois ne sont évidemment pas forcément choqués par ce paysage recréé parce que l'urbanisation, la construction de sites touristiques et la modification du paysage sont devenues chose courante en Chine. On entre ainsi dans la consommation du paysage via le produit; l'industrie touristique fait consommer le paysage autant que le thé et on consomme dans le paysage. 


\section{Compression du paysage}

46 production organise - produit - en même temps que certains rapports sociaux - son espace et son temps. C'est ainsi qu'il s'accomplit. » (Lefebvre, 1974, P. XXV.) Le concept de "Time-space compression », proposé par David Harvey dans son livre The Condition of Postmodernity pour décrire les caractéristiques spatiales du capitalisme contemporain, s'inscrit dans cette lecture. Selon Harvey, "l'espace semble se réduire à un "village global" de télécommunication et à une "terre spatiale" d'interdépendances économiques et écologiques, pour ne prendre que deux images familières et quotidiennes, et que les horizons temporels se raccourcissent à un point où le présent est tout ce qui existe (le monde du schizophrène), nous devons donc apprendre à faire face à un sentiment écrasant de compression de nos mondes spatial et temporel » (Harvey, 1989, p. 240).

Le paysage $d u$ thé, paysage ancien et paysage neuf inventé, peut figurer une des représentations concrètes de la "compression du temps et de l'espace ", puisqu'il reflète les transformations rapides de la Chine dans un contexte de libéralisme et partant les relations entre global et local ou entre le temps d'hier et celui d'aujourd'hui. En effet, les villes chinoises sont en train de développer en leur sein des paysages agricoles qui empruntent tant à la tradition qu'à la modernité. De telles évolutions gomment les changements saisonniers et décalages temporels : le paysage du thé tient du signe de la rencontre des temps et des espaces.

Dans les espaces de consommation des grandes villes, les éléments paysagers interviennent de plus en plus dans la décoration et l'ambiance, pour inciter à la consommation. Certains entrepreneurs de thé s'inspirent ainsi du modèle Starbucks, visant la clientèle en col blanc avec une consommation de thé instantané et de thé de fruit ou aux perles en gobelet à emporter. Il en est ainsi de la marque Heytea, née à Canton en 2012 et connue pour son thé de fromage (le fromage en poudre est ajouté dans le thé chinois). Elle a ouvert des boutiques de styles très variés dans les grandes villes, en espérant créer un «troisième espace » d'illusion : boutique Black décorée en noir et doré ; boutique Pink où le « jardin », les « feuilles d'arbres », le petit « chien », la cabine téléphonique, les tables et les chaises sont tous roses; boutique Day Dreamer Project avec la présence de la «montagne », du «cours d'eau » et de la "forêt », etc. ${ }^{13}$. Des éléments de paysage théicole deviennent ainsi décoration dans certains magasins avec de faux théiers et de faux rochers dans un style "naturaliste ». D'autres commerçants utilisent des images de théier artialisé sur leur panonceau (figures 29 et 30).

La «Petite boîte de thé » (xiaoguantea) est un autre exemple de compression où le paysage devient «capsule ». Son inventeur, Guofan Du, est un entrepreneur qui a investi dans de nombreux domaines (bandes correctives pour le dos, dictionnaires électroniques, tablettes et téléphones...) avant de créer cette petite boîte. L'emballage ressemble fortement à une capsule Nespresso et pour comparer son produit avec le thé traditionnel, Du évoque le costume occidental et le vêtement traditionnel chinois. La compression du paysage du thé atteint une sorte d'apogée via nos téléphones avec, par exemple, le tag wuyishan (shan qui signifie montagne en chinois) sur Instagram $^{14}$ : en trois mouvements sur un écran tactile, on accède à un paysage du thé encapsulé. 
50 De nouveaux modèles paysagers autour du thé circulent ainsi dans le monde, ces modèles étant tout à la fois profondément chinois mais aussi occidentaux. La mondialisation, l'urbanisation massive de la Chine s'expriment dans une hybridation de modèles et le paysage du thé, symbole intérieur et extérieur, en est une expression. Les formules diffusées empruntent tant au paysage traditionnel qu'à la modernité au point d'aboutir à un paysage du thé compressé, théâtral et simulé. Cette compression du paysage du thé, stade ultime de l'outil communicationnel paysage, multiplie les signes de connivence et, du point de vue touristique, s'impose comme formule à succès.

Figures 29. Boutique de thé aux monts Wuyi

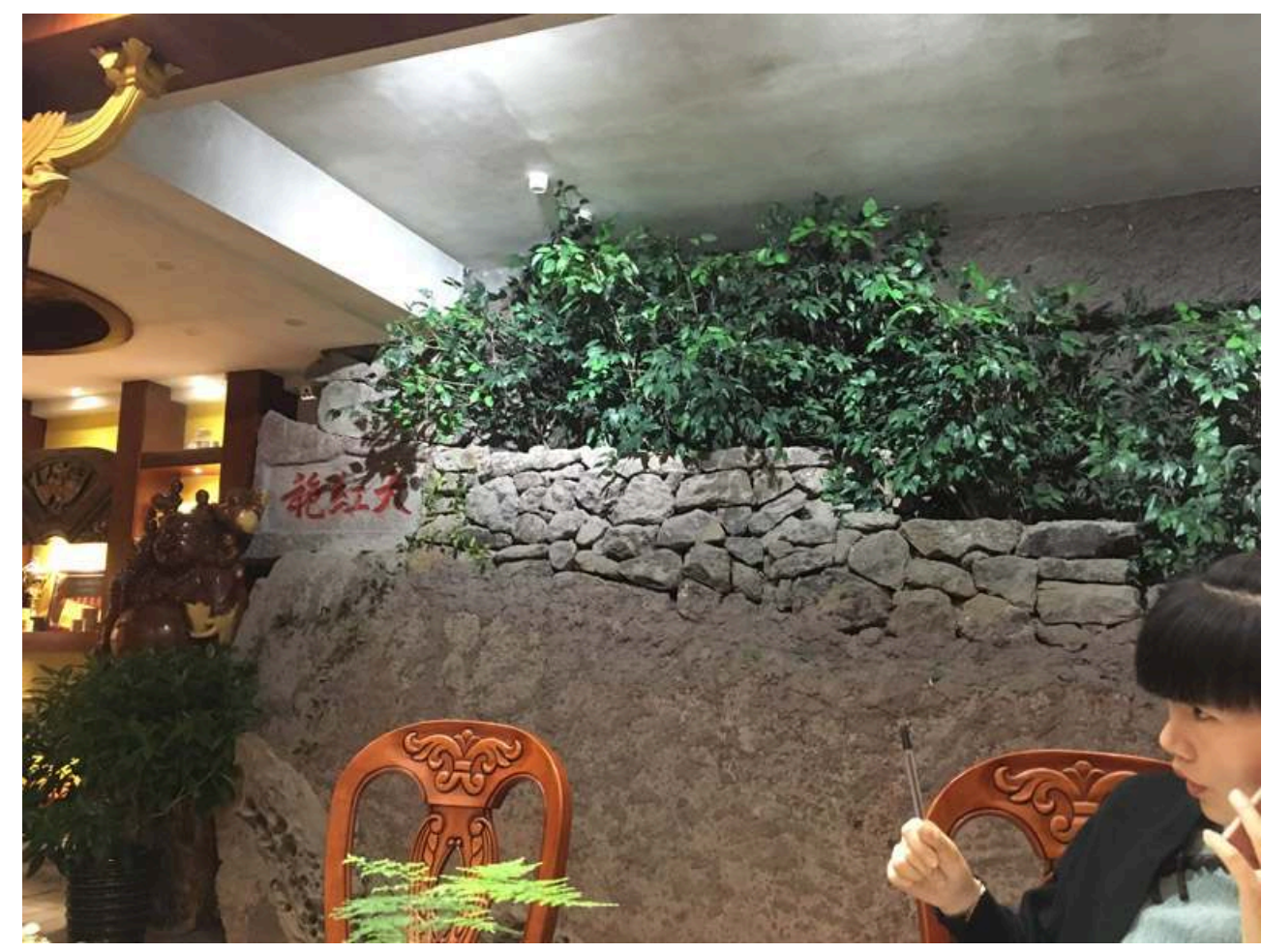

Photographiée en avril 2018. 
Figure 30. Boutique de thé aux monts Wuyi

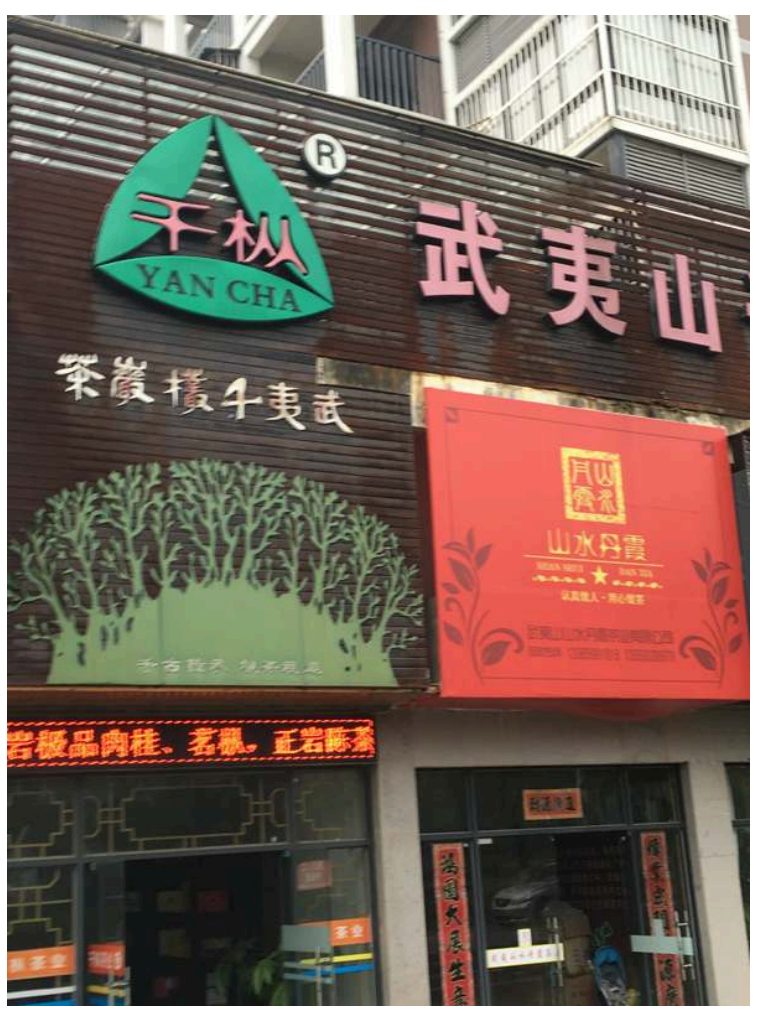

Photographiée en avril 2018.

\section{Conclusion}

51 Le paysage du thé en Chine est un paysage agricole avec des configurations spatiales complexes, mais qui évolue très lentement et présente de forts invariants sur la longue durée. Le vrai paysage du thé ne change pas ou peu à travers le temps, nonobstant des manières de cultiver qui intègrent certaines nouveautés (le rang par exemple). La mécanisation pourrait bouleverser la donne mais ce n'est pas une culture prioritaire en la matière. En revanche, la représentation de ce paysage a beaucoup évolué et bénéficie de manière originale d'apports tant chinois qu'occidentaux.

Il en est de même aujourd'hui où les publicités intègrent de plus en plus les vrais paysages du thé, d'autant que le grand public les connaît grâce au tourisme, aux documentaires, etc. Cette touristification se présente sous la forme d'un modèle international adapté à la chine où le paysage du thé, en tant qu'outil communicationnel, révèle des caractéristiques compressées et mêlées de temps et d'espace.

53 L'influence de la culture occidentale, dans la construction du paysage, les publicités chinoises, et le tourisme se lit fortement mais les éléments paysagers du thé sont interprétés et artificialisés, empreints de références occidentales, puis relocalisés en Chine. Si les Occidentaux ont toujours fantasmé l'exotisme de l'Orient, il existe également un regard oriental sur l'Occident. Bryan Turner souligne dans son livre Orientalism, Postmodernism and Globalism, que l'Orient et l'Occident ne sont pas de simples modèles dichotomiques et que le globalisme et le pluralisme pourraient 
remplacer l'orientalisme ou l'occidentalisme, en brisant les préjugés de la géopolitique et de la culture (Turner, 1994). Sous la vision du globalisme, les cultures chinoise et occidentale sont fluides et interactives. Ainsi si l'Occident a participé à la construction de l'image du paysage du thé chinois, il l'a fait aussi en digérant la culture du thé orientale aux XVIII et $\mathrm{XIX}^{\mathrm{e}}$ siècles pour l'intégrer à sa propre culture. La cup of tea est certes britannique, mais elle n'existerait pas sans la Chine, Robert Fortune et l'Inde.

Je remercie infiniment Monique Poulot pour son aide précieuse lors de la rédaction de cet article.

\section{BIBLIOGRAPHIE}

Le Secret du bon thé/好茶之道, Pékin, Triple Life Weekly/三联生活周刊, $\mathrm{n}^{\circ}$ 17, $\mathrm{n}^{\circ}$ 18, 2016, 2017.

Thé et Vin/茶酒二事, Pékin, Triple Life Weekly/三联生活周刊, $\mathrm{n}^{\circ} 16,2015$

Allom, T., China, In a Series of Views, Displaying the Scenery, Architecture, and Social Habits of that Ancient Empire, Londres, Fisher, Son, \& Co., 1843, vol. 2, 153 p.

Barthe, R., « Rhétorique de l'image », Communications, n 4, 1964, p. 40-51.

Baudrillard, J., La Société de consommation (1970), Paris, Gallimard, 1996, 320 p.

Berque, A., Les Raisons du paysage : de la Chine antique aux environnements de synthèse, Paris, Hazan, 1995, 192 p.

Berque, A., La Rizière et la Banquise : colonisation et changement culturel à Hokkaïdô, Paris, Publications orientalistes de France, 1980, 272 p.

Bouchery, P., « La rizière, "écosystème spécialisé" ? Un cas dans le Sud-Ouest chinois ", dans Arnoux, M., et Postel-Vinay, G. (dir.), Autres Temps, Autres Lieux. Études rurales, n 151-152, 1999, p. 133-165.

Chang, K., World tea production and trade Current and future development, Rome, FAO Intergovernmental Group on Tea, 2015, 13 p.

Chen, C., « Tea Classification in Theory and Practise ", Journal d'agriculture traditionnelle et de botanique appliquée, $\mathrm{n}^{\circ}$ 3-4, 1981, p. 329-344.

Chen, W., H., 陈文怀, « Discussion sur l'évolution et la classification des variétés du théier chinois/中国茶树品种演化和分类的商榷 》, Acta Horticulturalia Sinica/园艺学报, vol. 3, n² 2, 1964, p. 191-198.

Collectif, Larousse agricole. Le monde paysan au XXIe siècle, Paris, Larousse, 2002, 800 p.

Crang, M., Cultural Geography, Abingdon-on-Thames, Routledge, 1998, 224 p.

Darby, W. J., Landscape And Identity: Geographies of Nation And Class In England, Londres, Bloomsbury Academic, 2000, 330 p.

Deuss, J. J. B., « La culture et la fabrication du Thé », Journal d'agriculture traditionnelle et de botanique appliquée, vol. 5, n 4-5, 1958, p. 238-273. 
Deuss, J. J. B., « La Taille du théier en Indochine », Journal d'agriculture traditionnelle et de botanique appliquée, n 163, 1935, p. 153-170.

Du, Y., 杜育, Chuanfu/荈赋 (entre 265 et 317), Livre des Jin/晋书, 648.

Escande, Y., « Looking at a Landscape, an aesthetic approach/中西'景观'之观”的美学问题初探 》, Philosophie et culture/哲学与文化, vol. 39, n 11, 2012, p. 95-113.

Escande, Y., Montagnes et eaux : La culture du shanshui, Paris, Éditions Hermann, 2005, 293 p.

Escande, Y., « Paysage : regard croisé entre la peinture de paysage chinoise et la peinture de paysage occidentale I, II, III/景观 : 中国山水画与西方风景画的比较研究I、II、III », Vingt-etunième siècle/二十一世纪, n 78, 2003, p. 79-88., n 79, 2003, p. 77-86., nº 80, 2003, p. 101-105.

Fortune, R., Two Visits To The Tea Countries Of China China and the British Tea Plantations in the Himalaya, Londres, J. Murray, 1853, 352 p.

Fumey, G., « Paysages à boire et à manger », Les Carnets du paysage. Nourriture, n² 25, Arles/ Versailles, Actes Sud et l'École nationale supérieure de paysage, 2014, p. 53-63.

Fumey, G., Manger local, manger global, Paris, CNRS Édition, 2010, 160 p.

Goubeaux, M., J., « Observation sur la culture du théier en Indochine », Journal d'agriculture traditionnelle et de botanique appliquée, $\mathrm{n}^{\circ} 158,1934$, p. 865-873.

Guinard, A., « La culture du thé en Indochine », Archives des recherches agronomiques et pastorales au Viêtnam, $\mathrm{n}^{\circ} 20,1953,179 \mathrm{p}$.

Guo, Y., C., 郭元超, «Origine du théier/茶树原产考评 (续)》, Journal scientifique du thé/茶叶科学 简报, $\mathrm{n}^{\circ} 3,1988$, p. 13-17.

Harvey, D., The Condition of Postmodernity: An Enquiry into the Origins of Cultural Change, Hoboken, Wiley-Blackwell, 1989, $378 \mathrm{p}$.

Houssaye, J., G., Monographie du thé, Paris, Chez L'auteur, 1843, 160 p.

Jullien, F., Vivre de paysage ou l'Impensé de la raison, Paris, Gallimard, 2014, 258 p.

Kubin, W., Der durchsichtige Berg : die Entwicklung der Naturanschauung in der chinesischen Literatur, Stuttgart, F. Steiner Verlag Wiesbaden, 1985, 424 p., traduit en chinois en 1990 par Ma, S., D., Shanghai, Maison d'édition du peuple de Shanghai/上海人民出版社, 1990, 235 p.

Lefebvre, H., La Production de l'espace, Paris, Economica, 1974, 485 p.

Li, G. T., 李光涛, «L'étude sur l'ADN du théier et sa classification/茶树的核型及种的分类研究 », Thé/茶叶, nº 4, 1983, p. 11-16.

Li, J. Q., 李健权, Technologie pratique de la production du thé/茶叶优质高效生产实用技术, Hunan, Éditions Science et technologie de Hunan/湖南科学技术出版社, 2016, 106 p.

Lu, Y., « On David Harvey's Theory on Culture of Space », Journal of Guizhou university (Social Sciences), vol. 33, n 3, 2015, p. 137-143.

Lu, Y., 陆羽, Le Classique du thé/茶经 (780), traduit du chinois par C. Despeux., Paris, Rivage poche, 2015, p. 43-57.

Mendibil, D., «Publicité et Géographie : paysages, images et discours », Strates, n 4, 1989.

Métailié, G., « La ronde des thés », Terrain, n 13, 1989, p. 105-109, URL : http://

journals.openedition.org/terrain/2960. 
Min, Q.W., Zhang, Y.X., 闽庆文, 张永勋, Système de la culture du thé et du jasmin au Fuzhou/福建福 州茉莉花于茶文化系统, Pékin, Maison d'édition agricole de Chine/中国农业出版社, 2015, 256 p.

Onfray, M., La Raison gourmande. Philosophie du goût, Paris, Grasset, 1995, 254 p.

Sealy, J., R., A Revision of the Genus Camellia, Londres, Royal Horticultural Society, 1958, 239 p.

Trochain, J. « La production du thé et les améliorations apportées à la culture du théier en Indochine ", Journal d'agriculture traditionnelle et de botanique appliquée, nº 145, 1933, p. 613-650.

Turner, B., Orientalism, postmodernism and globalism, Abingdon-on-Thames, Routledge, 1994, 244 p.

Williamson, J., Decoding Advertisements, Ideology and Meaning in Advertising, Londres, Marion Boyars, 1978, $180 \mathrm{p}$.

Worswick, C., Spence, J., Imperial China : Photographs 1850-1912 (1978), Canberra, Australian National University Press, 1980, 151 p.

Wu, J. N., 吴觉农, Exégèse du classique du thé/茶经述评, Pékin, Maison d'édition agricole de Chine/ 中国农业出版社, 2005, 369 p.

Wue, R., « China in the World: On Photography, Montages, and the Magic Lantern », History of Photography, vol. 41, 2017, p. 171-187.

Xiao, K. B., 肖坤冰, Flux de thé-La narration matérielle, spatiale et historique des régions montagneuses $d u$ Fujian (1644-1949)/茶叶的流动:闽北山区的物质、空间与历史叙事(1644-1949), Pékin, Édition Université de Pékin/北京大学出版社, 2013, 239 p.

Xu, C., S., 许次纾, Chashu/茶疏 (1597), Siku Quanshu/四库全书, 1782, https://zh.wikisource.org/ wiki/茶疏

Zhang, H., D., 张宏达, «Ajout au système de classification du théier/茶叶植物资源订正 》, Journal de l'Université de Zhongshan/中山大学学报：自然科学版, n 1, 1984, p. 1-25.

Zhang, Y. Y., 张彦远, Le Lidai Ming Hua Ji/ 历代名画记 (entre 847 et 859), Pékin, Maison d'édition des beaux-arts du peuple/人民美术出版社 2004, 205 p.

\section{NOTES}

1. La Chine a produit 1,9 million de tonnes de thé en 2013 , soit $38 \%$ de la production mondiale. Source : Chang, 2015.

2. Les dates retenues sont avant tout celles de notre corpus; nous les mettrons en relation avec les grandes dates de l'histoire chinoise.

3. Un pied, mesure chinoise traditionnelle, équivaut $30.6 \mathrm{~cm}$.

4. En chinois l'expression " jardin du thé » désigne une exploitation de thé.

5. Le plus ancien traité du paysage, Introduction à la peinture de paysage, a été rédigé par un peintre chinois, Bing Zong.

6. Zhang, Y.Y., 张彦远, Le Lidai Ming Hua Ji, vol. 6. Dans cette citation, Zhang fait référence à Bing Zong. «WoYou » (Voyage couché), proposé par Bing Zong : le transport était incommode, et les lettrés utilisaient donc la peinture pour apprécier le paysage.

7. Certaines sont de libre de droits.

8. Le mouvement d'auto-renforcement, entre 1861 et 1895 , est un mouvement de réformes institutionnelles en Chine.

9. Selon Clark Worswick, très peu de photographes chinois au XIX ${ }^{\mathrm{e}}$ siècle ont pris des photos des paysages extérieurs (Worswick, 1978, p. 144). Robert Wue souligne que les photos de portraits 
sont dominantes à cette époque chez les photographes chinois (Wue, 2017, p. 171-187). Le deuxième voyage du sinologue Édouard Chavannes au mont Tai en 1907 est un cas particulier. Assisté par un photographe chinois, Zhou, ils ont photographié ensemble des paysages.

10. Voir les photos sur le lien : http://www.tuanjiebao.com/lishi/2017-08/23/ content_116889.htm.

11. Les distributeurs de thé ouvrent au $\mathrm{XX}^{\mathrm{e}}$ siècle de grands magasins de thé et des salons, souvent pour la vente au détail. Le contact avec l'agriculture est rare et les consommateurs sont surtout attachés aux vertus du produit.

12. «Le village caractéristique chinois » est un plan exécuté par la commission nationale pour le Développement et la Réforme, le ministère des Finances et le ministère du Logement et du Développement urbain-rural, pour développer les petites villes et villages en Chine. D'ici à 2020, il est prévu d'inscrire environ 1000 villages caractéristiques mais la ville de Wuyishan n'en fait pas partie.

13. Voir les photos sur le lien : http://chuangkr.china.com.cn/p/5114938?column=BUSINESS.

14. https://www.instagram.com/explore/tags/wuyishan/.

\section{RÉSUMÉS}

À partir d'un travail documentaire et d'enquête sur deux zones autour des villes de la province du Fujian (monts Wuyi et Fuzhou), l'article envisage la genèse du paysage du thé et de son image. Ce paysage du thé, né de la domestication de plantes sauvages et qui a donné lieu à nombreuses représentations (matérielles et immatérielle), est le fruit d'une construction d'abord orientale, mais avec un apport important de l'Occident. L'article s'attache également à caractériser l'utilisation de l'image du thé et de son paysage dans la publicité, en se focalisant sur plusieurs nœuds temporels (fin $\mathrm{du} \mathrm{XIX}^{\mathrm{e}}$ siècle, début $\mathrm{du} \mathrm{XX}^{\mathrm{e}}$ et période postérieure aux réformes économiques des années 1980 en Chine), et à étudier d'autres constructions autour du paysage du thé à visée publicitaire. Il s'agit ainsi de comprendre comment et sous quelle forme le paysage du thé, construit dans la durée par ces multiples représentations, est aujourd'hui consommé.

Based on documentary work and a survey of two areas around the cities of Fujian Province (Wuyi Mountains and Fuzhou), the article considers the genesis of the tea-growing landscape and its image. This tea-growing landscape, born of the domestication of wild plants and which is at the origin of many tangible and intangible representations, is not only an oriental construction, it is also the result of many contributions from the West. This article describes the use of the image of tea and its landscape in advertising, focusing on several periods (the late 19th century, early 20th century and the period following the economic reforms in China in the 1980s). Also studied are other images based on the tea-growing landscape created for the purpose of advertising. The objective is to understand how and in what form the tea-growing landscape, which has been built over time by these representations, is consumed today.

\section{INDEX}

Mots-clés : paysage du thé, image paysagère, publicité, consommation, mondialisation

Keywords : tea-growing landscape, landscape image, advertising, consumption, globalisation 


\section{AUTEUR}

\section{YUN ZHANG}

Yun Zhang, architecte-paysagiste, est diplômée en paysagisme et architecture (université de sylviculture de Nankin en Chine) et d'un master en paysagisme (École nationale supérieure de paysage de Versailles). Actuellement doctorante en géographie et paysagisme à l'université Paris Nanterre, elle prépare une thèse intitulée « Paysages du thé, paysages viticoles : approches croisées en Fujian et en Bourgogne » sous la direction de Monique Poulot au laboratoire LavueMosaïques.

yunzhang0123[at]gmail[dot]com 